\title{
A DOMAIN-THEORETIC APPROACH TO INTEGRATION IN HAUSDORFF SPACES.
}

\section{J. D. HOWROYD}

\begin{abstract}
In this paper we generalize the construction of a domain-theoretic integral, introduced by Professor Abbas Edalat, in locally compact separable Hausdorff spaces, to general Hausdorff spaces embedded in a domain. Our main example of such spaces comprises general metric spaces embedded in the rounded ideal completion of the partially ordered set of formal balls. We go on to discuss analytic subsets of a general Hausdorff space, and give a sufficient condition for a measure supported on an analytic set to be approximated by a sequence of simple valuations. In particular, this condition is always satisfied in a metric space embedded in the rounded ideal completion of its formal ball space. We finish with a comments section, where we highlight some potential areas for future research and discuss some questions of computability.
\end{abstract}

\section{Introduction}

The aim of this paper is to provide a general domain-theoretic construction of an integral akin to the classical Riemann integral on compact intervals of the real line. Such an integral was first constructed in compact metric spaces in [3] and generalized to locally compact separable Hausdorff spaces in [12]. The constructions in $[3,12]$ begin by embedding the Hausdorff space into an associated domain - the upper space ordered by reverse inclusion; see Subsection 2.2 for a definition. Here we generalize this approach to an arbitrary Hausdorff space (topologically) embedded into a domain; in particular, any metric space may be embedded into the rounded ideal completion of its formal ball space. When this general framework has been set up, the definition of the integral in [3] carries over directly to our setting; the main work is then to show that the properties of the integral carry over to our framework. We, therefore, propose the name Riemann-Edalat integral for the construction that we give.

The integral is with respect to a $\mathcal{T}$-continuous probability Borel measure on the Hausdorff space. This gives rise to a continuous valuation on the domain, which may be approximated by simple valuations. Corresponding to each simple valuation are upper and lower Darboux sums that bound the value of the integral. The integral is then defined by taking the 'limit' of these Darboux sums over the directed family of approximating simple valuations. In this way, it is the measure that is approximated by simple valuations, as opposed to the construction of the Lebesgue integral where the function to be integrated is approximated by simple functions. 
We go on to study the properties of the Riemann-Edalat integral, and compare it with the Lebesgue integral. Our results, in this respect, are analogous to the standard results concerning the Riemann integral. Similar results were shown in $[3,12]$; however, new proofs of our main results, Theorem 12 and Proposition 14, are required for our framework. We also mention that if the underlying space is completely regular (and Hausdorff) then the Bourbaki extension of the Riemann-Edalat integral recaptures the Lebesgue integral. We then proceed with an investigation into integration over subsets of the underlying space of a certain form; namely $D$-Suslin sets. This leads to a construction of a sequence of simple valuations, approximating the measure $\mu$, which is sufficient to compute the integral with respect to a measure supported on such a set. The notion of a $D$-Suslin set is closely related to the notion of an analytic set, and indeed in a metric space (embedded in its associated domain) they are equivalent. We conclude with a comments section, where we remark on a number of alternatives and some topics for further attention.

\subsection{Motivation}

Domain theory, introduced by Dana Scott in 1970, has become a basic paradigm in theoretical computer science; in particular, as a mathematical foundation for denotational semantics. The success of this theory has been in using domains as a model for a computational process or as a model for a data type with incompletely specified elements. A new area of research has focused on using domain theory for computational models of spaces in classical mathematics. The survey paper [7] gives a comprehensive overview of these developments. We give a selective overview in the next paragraph of some of these developments, which are of particular interest in the context of this paper.

We begin with [5], which presented a computational model (namely, the upper space ordered by reverse inclusion) for locally compact second countable Hausdorff spaces. This gave a computational framework in which dynamical systems, iterated function systems and measure theory could be discussed. This was set up in [5] and extended in $[\mathbf{8}]$; also see $[\mathbf{6 , 1 3}]$ for further applications to iterated function systems. The framework for measure theory was based on the foundational work concerning valuations on a domain given in [19, 22]; see also [17]. This led on to the development in [3] of a domain-theoretic integral on compact metric spaces, which was generalized to locally compact second countable Hausdorff spaces in [12]. Applications of this framework have been given, for example, in stochastic processes (see [4]) and in statistical physics (see [2]). Then in [10] a new computational model (namely, the ideal completion of the space of formal balls) was presented for metric spaces, giving a framework for iterated function systems and measure theory in this setting; see [18] for further developments concerning this model. Also, see $[\mathbf{2 4}, \mathbf{2 5}]$ for a detailed discussion of computational models for polish spaces. In a slightly different direction, we mention the treatment of computability (of points, sequences and functions) in the real line given in [14], using the interval domain (see Subsection 2.2) as a computational model for the real line. This work was then extended in [15] to a treatment of computability in Banach spaces, using the model of [10]. We also mention the ripening theory of exact real number arithmetic; in connection with integration theory, see $[\mathbf{9 , 1 1}]$.

By taking the model of [10] as our main example (of a Hausdorff space embedded in a domain), we give, in this paper, a domain-theoretic integral in this setting. We also remark that the spaces on which the domain-theoretic integral was constructed in $[3,12]$ are by hypothesis metrisable. Thus, if the metric of these spaces is known, then the computational model of [10] may be used to construct the integral. On the other hand, in other circumstances 
a new domain-theoretic model may better reflect the underlying structure of the Hausdorff space in question. In this respect we develop a general theory that is applicable whenever a Hausdorff space is embedded in a domain.

There are various theories of integration in mathematics. Perhaps the most elementary is that of reverse differentiation, which dates back to the time of Newton. However, some functions $f: \mathbb{R} \rightarrow \mathbb{R}$, most notably $f(x)=e^{-x^{2}}$, could not be integrated by this method, yet their integral seemed to make sense. The problem is to write down a function $F$ in terms of a list of standard functions which differentiates to give $f$. The introduction of Riemann integration by mathematicians such as Cauchy, Riemann, Stieltjes and Darboux gave the first cohesive mathematical theory. These mathematicians could say that $e^{-x^{2}}$ is (Riemann) integrable (on compact intervals) and that there is a function $F$ whose derivative is $e^{-x^{2}}$ but it may be not possible to write it down in terms of the standard list of functions of their time; this was later shown to be the case. It was also possible to integrate some functions where the indefinite integral is not differentiable (at every point). An important part of the Riemann theory of integration is its constructive nature, which has led to the development of numerical methods to approximate the value of an integral.

A significant limitation to the theory of Riemann integration is that it lacks good convergence properties. One way that this limitation was overcome was by extending the Riemann integral by defining the integral of a function which is the monotone limit of a sequence of integrable functions. This is the basis upon which the Daniell theory of integration arose. At about the same time Lebesgue, among others, developed a new theory of integration, which also had good convergence properties. It turns out that the Lebesgue integrable functions (with respect to the standard Lebesgue measure) are precisely those that are Daniell integrable. Similarly, those functions which are integrable with respect to the Daniell extension of the Riemann-Stieltjes integral are precisely those that are Lebesgue-Stieltjes integrable. However, the Lebesgue integral could be defined in abstract spaces whenever a measure is given on the abstract space. With this advantage, the Lebesgue theory stands as a basis for modern integration theory and analysis.

Further developments include the McShane integral, which is equivalent to the LebesgueStieltjes integral, and the theories of Denjoy, Perron and Henstock, which are all equivalent to each other, and numerous other variations on these themes, giving generalizations in various ways. Developments in other directions include contour integration of complex analysis, de Rahm cohomology, stochastic calculus (in particular Ito integrals and Stratonovich integrals), Feinman integrals, and so on. However, much of the constructive nature of the Riemann integral is lost from these general theories.

The theory of integration that we present here keeps the constructive nature of the Riemann integral, and indeed naturally leads to algorithms that can evaluate an integral (of a Riemann-Edalat integrable function) to any desired accuracy. In contrast to the Riemann integral, we are not limited to integration only in the Euclidean spaces $\left(\mathbb{R}^{n}\right)$, our construction being applicable in many abstract spaces. Every Riemann-Edalat integrable function is Lebesgue integrable, and the values of the integrals agree, see Subsection 4.3. In fact, a Riemann-Edalat integrable function must be bounded and continuous almost everywhere which, in general, is a more restrictive condition than Lebesgue summability. However, in the case when the underlying space is completely regular (and Hausdorff), the Bourbaki extension of the Riemann-Edalat integral (from the space of continuous functions) recaptures the Lebesgue integral, see Subsection 4.4 for details. In Subsection 7.4, we discuss another extension process which is appropriate when the underlying space is not completely 
regular, this time, the extension based on the integral of a continuous function on a domain with respect to a continuous valuation.

Thus our theory of Riemann-Edalat integration should not be seen as a more general integral than, say the Lebesgue integral, but as an extension of the Riemann integral to more abstract spaces - giving a computational framework to integration in this setting. In application, this gives a method, which may be encoded in a computer program, to evaluate specific integrals of physical or theoretical interest where traditional numerical methods are inappropriate. Secondly, our domain-theoretic integral should provide a means by which to answer theoretical questions of computability; for example, what is the class of functions for which the integral with respect to a given measure is computable. Lastly, it is also hoped that there may be some feedback into the classical theory of integration and measure which may lead to new and interesting results.

\subsection{Outline of the paper}

We now give a section-by-section outline of this paper. We follow the introduction by outlining the basic set-up to be considered throughout the rest of the paper, and give definitions of the notation and terminology used. We also give, in Subsection 2.2, a number of examples of constructions of a domain into which a particular type of Hausdorff space may be embedded; in particular, the rounded ideal completion of the partially ordered set of formal balls of a general metric space, introduced and discussed in [10]. This example was the main driving force in the construction given in this paper of the domain-theoretic integral, and thus may provide an intuitive insight into the proofs of the results that follow.

Section 3 gives the definition of the integral, with respect to a $\mathcal{T}$-continuous probability Borel measure, and shows its basic properties. In particular, Corollary 9 asserts that $\mathcal{R}(\mu)$, the family of $\mu$-integrable functions, is a real vector space, and that integration, with respect to $\mu$, is a positive linear functional. This section is based on the work presented in [3].

The next section goes on to explore the properties of this integral. We mention Theorem 12 , which characterizes the family $\mathcal{R}(\mu)$ of all $\mu$-integrable functions, and the discussion in Subsection 4.4 of the Bourbaki extension of our integral in the case when the underlying space $X$ is completely regular and Hausdorff. We also outline, in this case, how the Bourbaki integral recaptures the measure $\mu$ and the Lebesgue integral with respect to $\mu$.

In Section 5 we introduce a concept of a $D$-Suslin subset. We then give, for a $\mathcal{T}$-continuous probability Borel measure $\mu$ supported on a $D$-Suslin subset $A$, a construction of a sequence $\left(v_{n}\right)_{n=0}^{\infty}$ of approximating simple valuations with $\hat{\mu}=\bigsqcup_{n=0}^{\infty} v_{n}$. In particular, the (upper and lower) integrals of a function $f: X \rightarrow \mathbb{R}$ may be evaluated from the (upper and lower) Darboux sums corresponding to each of the simple valuations in $\left(v_{n}\right)_{n=0}^{\infty}$. Furthermore, if $\mu$ is any $\mathcal{T}$-continuous Borel measure on the underlying Hausdorff space $X$, then this directly leads to a definition of the integral $\int_{A} f \mathrm{~d} \mu$ of a function $f: X \rightarrow \mathbb{R}$ over any $D$-Suslin subset $A$ of $X$ with $0<\mu(A)<\infty$. Details of this may be found in the introductory comments for the section.

Section 6 gives a sufficient condition for an analytic subset of a general Hausdorff space $X$, embedded in the domain $D$, to be $D$-Suslin. This is done using the notion of a countable graded cover. It is also shown that in the case when $X$ is a metric space and $D$ is the rounded ideal completion of the space of formal balls then this condition is met, and hence every analytic subset is $D$-Suslin. We end this section with a discussion relating these ideas to the support of a $\mathcal{T}$-continuous probability Borel measure on a complete metric space.

Finally, we comment, in Section 7 , on a number of points. We start by mentioning a 
few drawbacks of the current theory. Next we remark that there is some latitude in our definition of the integral; specifically, the functions $f_{*}$ and $f^{*}$ may be changed. However, the functions $f_{*}$ and $f^{*}$ are precisely the functions that make our definition of the integral logically equivalent to the definition of the integral in $[3,12]$ on compact and locally compact separable Hausdorff spaces. Subsection 7.3 lists a number of questions concerning topological measure theory that have arisen within this paper. In Subsection 7.4 we introduce an extension process of the integral on a domain $D$ with respect to continuous valuations, giving a new integral on the underlying space $X$, which we suggest recaptures the Lebesgue integral of a function with respect to a $\mathcal{T}$-continuous locally finite regular Borel measure on $X$. If this integral could be placed in a computable framework then we feel that it would be an excellent field for future research. We finish the section with some comments concerning some of the many questions of computability with respect to the work described within this paper.

\subsection{Acknowledgements}

Professor Abbas Edalat inspired much of the worked presented here, with many discussions that we had together. Mauricio Alvarez-Manilla pointed out that the assumption throughout an earlier manuscript that all measures were $\mathcal{T}$-regular was unnecessary. Thanks are also given to the anonymous referees for their numerous comments and corrections.

\section{Preliminaries}

In this section we set up the basic definitions, terminology and constructions that will be used throughout the rest of this paper.

\subsection{The underlying spaces}

Throughout this paper $X$ will be a space of points endowed with a Hausdorff topology $\mathcal{T}$, and $D$ will be a domain with ordering $\sqsubseteq$ and a least element $\perp$; by a domain we mean a continuous directed complete partially ordered set. We use the standard terminology and notations of domain theory, as for example in [1], and of topology, as for example in [21]. We also suppose that $e: X \rightarrow D$ is a topological embedding where $D$ is given the Scott topology. In many circumstances $X$ is embedded as (a subset of) the space of maximal points of $D$. However, in this paper, we shall not assume this; an example of one of the anonymous referees shows that some generality is gained by not making this assumption.

We recall that a non-empty subset $A \subseteq D$ is upwardly directed whenever for all $a, b \in A$ there exists $c \in A$ with $a, b \sqsubseteq c$. By saying that $D$ is directed complete we mean that every upwardly directed set $A$ has a supremum, which we denote by $\bigsqcup A$. For $c, d \in D$, we say that $c$ approximates $d$ and write $c \ll d$ whenever for all upwardly directed subsets $A \subseteq D$ with $d \sqsubseteq \bigsqcup A$ there exists $a \in A$ with $c \sqsubseteq a$. We say that a subset $B \subseteq D$ is a basis of approximation of $D$ whenever for every element $d \in D$ the set $B_{d}=\{b \in B \mid b \ll d\}$ is an upwardly directed set with $d=\bigsqcup B_{d}$. A directed complete partially ordered set is said to be continuous when it has a basis of approximation. A subset $C \subseteq D$ is a lower set whenever for all $d \in D$ with $d \sqsubseteq c$ for some $c \in C$ we have $d \in C$. Finally, the Scott topology is defined by giving the closed sets as those sets $C \subseteq D$ which are lower sets and closed under the supremum of directed sets; that is, for all upwardly directed sets $A \subseteq C$ we have $\bigsqcup A \in C$.

The embedding $e$ allows us to define two set-valued maps, $[\cdot]: D \rightarrow \mathcal{P}(X)$ by $[d]=$ 
$e^{-1}(d \uparrow)$ and $(\cdot): D \rightarrow \mathcal{T}$ by $(d)=e^{-1}(d \uparrow)$, where

$$
d \uparrow=\{c \in D \mid d \sqsubseteq c\} \quad \text { and } \quad d \uparrow=\{c \in D \mid d \ll c\} .
$$

We remark that an alternative, leading to a slightly different theory, would be to define $[d]$ as the topological closure (in $X$ ) of $e^{-1}(d \uparrow)$; however, we shall not pursue this here.

Note 1. We observe the following simple consequences of these definitions.

1. If $a \subseteq b$ then $[b] \subseteq[a]$ and $(b) \subseteq(a)$.

2. If $a \ll b$ then $[b] \subseteq(a)$, and $[b]=\bigcap_{a \ll b}(a)$.

3. $[\perp]=(\perp)=X$.

4. If $B \subseteq D$ is a basis of approximation, then $\{(b) \mid b \in B\}$ is a basis of the topology $\mathcal{T}$.

5. It follows from the fact that $\mathcal{T}$ is Hausdorff that $[e(x)]=\{x\}$ for all $x \in X$.

We use $\mathbb{R}$ to denote the real numbers, together with the usual operations of addition, multiplication and order $\leqslant$. The positive reals $(x \in \mathbb{R}$ with $x>0)$ will be denoted by $\mathbb{R}^{+}$, the non-negative reals by $\mathbb{R}_{0}^{+}$, the extended reals $(\mathbb{R} \cup\{-\infty, \infty\})$ by $\overline{\mathbb{R}}$, and the non-negative extended reals $\left(\mathbb{R}_{0}^{+} \cup\{\infty\}\right)$ by $\overline{\mathbb{R}}_{0}^{+}$, with the usual conventions concerning $\pm \infty$. We shall, however, state explicitly each time that we require the convention

$$
\pm \infty \cdot 0=0=0 \cdot \pm \infty .
$$

For real-valued functions, with a common domain, the algebraic operations, partial ordering $\leqslant$, and suprema and infima are defined pointwise. We use $f \wedge g$ for $\min \{f, g\}$, and $f \vee g$ for $\max \{f, g\}$. Also, $f^{+}$will denote $f \vee 0$, and $f^{-}$will denote $-(f \wedge 0)$. A non-empty family $\mathcal{F}$ of real-valued functions, with a common domain, is said to be upwardly directed whenever for each $f, g \in \mathcal{F}$ there exists $h \in \mathcal{F}$ with $f \vee g \leqslant h$. We write $\mathcal{F} \nearrow g$ when $\mathcal{F}$ is upwardly directed and $g=\sup _{f \in \mathcal{F}} f$. Dually, we define downwardly directed and $\mathcal{F} \searrow g$. For $E \subseteq X$ we denote the characteristic function of $E$ by $\mathbb{1}_{E}$; that is, $\mathbb{1}_{E}: X \rightarrow \mathbb{R}$ and is defined by

$$
\mathbb{1}_{E}(x)= \begin{cases}1 & \text { if } x \in E \\ 0 & \text { otherwise }\end{cases}
$$

\subsection{Examples of such spaces}

The first example that we consider is when $X$ is a locally compact (Hausdorff) space. We define the upper space $\mathbf{U}_{\perp} X$ to be the space

$$
\mathbf{U}_{\perp} X=\{K \subseteq X \mid K \text { is non-empty and compact }\} \cup\{X\},
$$

ordered by reverse inclusion $\sqsubseteq=\supseteq$, which is seen to be a domain with least element $X$. The embedding $e: X \rightarrow \mathbf{U}_{\perp} X$ is defined for $x \in X$ by $e(x)=\{x\}$. This construction was discussed extensively in [5], and a theory of integration was discussed in [3] in the case that $X$ is compact and metrisable (and hence also separable), and in [12] in the case that $X$ is locally compact and separable. The theory of integration discussed in $[3,12]$ is easily seen to be entirely equivalent to the theory discussed here with $D=\mathbf{U}_{\perp} X$. Note, however, that here we do not assume that the space is separable.

Another example is given by the interval domain $\mathbf{I}_{\perp} \mathbb{R}$ of $\mathbb{R}$ defined by

$$
\mathbf{I}_{\perp} \mathbb{R}=\{[\alpha, \beta] \mid \alpha, \beta \in \mathbb{R} \text { and } \alpha \leqslant \beta\} \cup\{\mathbb{R}\},
$$

and ordered by reverse inclusion. Again, $\mathbf{I}_{\perp} \mathbb{R}$ is a domain, with least element $\mathbb{R}$, and the embedding $e: \mathbb{R} \rightarrow \mathbf{I}_{\perp} \mathbb{R}$ is defined for $x \in \mathbb{R}$ by $e(x)=[x, x]=\{x\}$. See [14] for an 
approach applying this domain to computability on the real line. We also mention [9], which develops integration in Real PCF; here it is the interval domain $\mathbf{I}[0,1]$ that is of interest, defined by

$$
\mathbf{I}[0,1]=\{[\alpha, \beta] \mid \alpha, \beta \in \mathbb{R} \text { and } 0 \leqslant \alpha \leqslant \beta \leqslant 1\}
$$

and ordered, as above, by reverse inclusion.

The main example of the paper is when $X$ is a metric space with metric $\rho$ and $D$ is the space $\mathcal{J}(\mathbf{B} X)_{\perp}$ to be defined in the next paragraph. Indeed the two (related) notions of 'fine covering system', see Subsection 4.2, and of 'graded covering', see Subsection 6.2, were generalizations of constructions using formal balls. The space $\mathbf{B} X$ is the set $X \times \mathbb{R}_{0}^{+}$ with the ordering $\sqsubseteq$, defined by $(x, r) \sqsubseteq(y, s)$ whenever $(r \geqslant s$ and $) \rho(x, y) \leqslant r-s$. Note that there is a natural topological embedding (with respect to the Scott topology on $\mathbf{B} X) \iota: X \rightarrow \mathbf{B} X$ defined by $\iota(x)=(x, 0)$. This construction was discussed extensively in [10] and further discussed in [18]. In the next paragraph we summarize the main results from [10] that we shall use.

The space $\mathbf{B} X$ is a domain if and only if $X$ is complete. In the case that $X$ is not complete, $\mathbf{B} X$ is a partially ordered set on which, although it is not directed complete, a 'way below' relation « can be defined in the usual way; $a \ll b$ whenever for all directed sets $B \subseteq \mathbf{B} X$ with least upper bound greater than $b$, some element of $B$ is greater than $a$. This definition amounts to $(x, r) \ll(y, s)$ whenever $(r>s$ and) $\rho(x, y)<r-s$. The space $\mathbf{B} X$ with the relation $\ll$ is an abstract basis, and hence, as in [1], we can 'construct' the rounded ideal completion. A subset $I \subseteq \mathbf{B} X$ is a rounded ideal (or «-ideal) whenever $I$ is a (non-empty) upwardly directed, lower set with respect to the relation $\ll$. The space $\mathcal{J}(\mathbf{B} X)$ is the family of all rounded ideals $I \subseteq \mathbf{B} X$ ordered by set inclusion. By adjoining a bottom element $\perp$, which we can take to be $\emptyset$, we obtain the space $\mathcal{J}(\mathbf{B} X)_{\perp}$. By [1, Proposition 2.2.22], $\mathcal{J}(\mathbf{B} X)_{\perp}$ is a domain, with $\downarrow: \mathbf{B} X \rightarrow \mathcal{J}(\mathbf{B} X)_{\perp}$ a natural (continuous) embedding. We note that $e=\Downarrow \circ \iota: X \rightarrow \mathcal{J}(\mathbf{B} X)_{\perp}$ is a topological embedding such that

$$
e(X)=\Downarrow \circ \iota(X)=\{(x, 0) \Downarrow \mid x \in X\}
$$

is contained in the space of maximal points (which is homeomorphic to the topological completion of $X$ ). We also note that in the case that $X$ is a complete metric space we have

$$
D=\mathcal{J}(\mathbf{B} X)_{\perp} \cong \mathbf{B}_{\perp} X=\mathbf{B} X \cup\{\perp\} .
$$

We remark that if $X$ is a second countable locally compact space, or if $X$ is a complete separable metric space (and thus second countable), then in the above constructions the resulting domain $D$ has a countable basis of approximation and $e(X)$ is the space of maximal points of $D$. Also, in these cases, for all $d \in D$ we see that $[d]$ is closed in $X$, and consequently that on $e(X)$ the inherited Scott topology and the inherited Lawson topology coincide. It follows by [24, Corollary 2.5] that $e(X)$ is a $G_{\delta}$ subset of $D$ in the Scott topology (that is, $e(X)$ is a countable intersection of Scott open sets), and by [24, Corollary 2.6] that $e(X)$ is polish; in particular $X$ is metrisable. A number of useful consequences of this setup are given in [8] and in [25]. We mention [25, Theorem 8.7], which asserts that the map $\mu \mapsto \hat{\mu}$ (to be defined in Subsection 2.4) is a homeomorphism between the space $\mathbf{M}^{1} X$ of probability Borel measures on $X$ with the weak topology and the space of maximal points $\operatorname{Max}\left(\mathrm{P}^{1} D\right)$ of the normalised probabilistic power domain $\mathrm{P}^{1} D$ with the inherited Scott topology. This result follows on from the work presented in [8], where it is shown, in [8, Proposition 4.2 and Corollary 4.1], that whenever $X$ is a separable metric space embedded as a $G_{\delta}$ subset of $D$, and consequently a subset of the space of maximal points, then the 
map $\mu \mapsto \hat{\mu}$ is an embedding of $\mathbf{M}^{1} X$ into a subset of the space of maximal points of the probabilistic power domain $\mathbf{P} D$.

\subsection{Measures on $X$}

Throughout this paper we will be concerned with measures $\mu$ on $X$, which in our nomenclature will be termed $\mathcal{T}$-continuous probability Borel measures. All measures $\mu$ on $X$ will be assumed to be outer measures; that is, $\mu: \mathcal{P}(X) \rightarrow \overline{\mathbb{R}}_{0}^{+}$is a function satisfying the following conditions:

1. $\mu(\emptyset)=0$;

2. if $E, F \subseteq X$ with $E \subseteq F$ then $\mu(E) \leqslant \mu(F)$;

3. for any sequence $\left(E_{i}\right)_{i=1}^{\infty}$ of subsets of $X$ we have $\mu\left(\bigcup_{i=1}^{\infty} E_{i}\right) \leqslant \sum_{i=1}^{\infty} \mu\left(E_{i}\right)$.

If $\mu(X)<\infty$ then $\mu$ is said to be finite, and if $\mu(X)=1$ then $\mu$ is said to be a probability measure. The advantage of the outer measure theory is that we do not have to worry about whether the measure of a given set is defined; however, we will need to know which sets are 'measurable'. We recall Carathéodory's criterion: a set $M \subseteq X$ is said to be $\mu$-measurable if for all $E \subseteq X$ we have

$$
\mu(E)=\mu(E \cap M)+\mu(E \backslash M) .
$$

We denote by $\mathcal{M}_{\mu}$ the family of all $\mu$-measurable sets which, as is well known (see, for example, [27, Theorem 2]), is a $\sigma$-algebra; that is, $\mathcal{M}_{\mu}$ is closed under the set operations of complement and countable union.

We let $\mathcal{B}$ denote the family of all Borel subsets of $X$ which is the smallest $\sigma$-algebra that contains the family $\mathcal{T}$ of all open subsets of $X$; equivalently, $\mathcal{B}$ is the smallest family of subsets of $X$ that contains the family of all open sets and the family of all closed sets, and is closed under countable unions and (non-empty) countable intersections. A measure $\mu$ is a Borel measure whenever $\mathcal{B} \subseteq \mathcal{M}_{\mu}$ and $\mu$ is $\mathcal{B}$-regular (which is often referred to as Borel regular); namely, for all $E \subseteq X$ we have

$$
\mu(E)=\inf \{\mu(B) \mid E \subseteq B \in \mathcal{B}\} .
$$

The more conventional notion of a Borel measure is of a countably additive set function $\nu: \mathcal{B} \rightarrow \overline{\mathbb{R}}$. We may define the (outer) measure extension $\mu$ of $\nu$ by

$$
\mu(E)=\inf \{v(B) \mid E \subseteq B \in \mathcal{B}\}
$$

for all $E \subseteq X$. Then $\mu$ is a Borel (outer) measure, in particular $\mathcal{B} \subseteq \mathcal{M}_{\mu}$, that agrees with $v$ on $\mathcal{B}$; see [27, Section 2.4] or [26, exercises 12-3 through to 12-11]. Moreover, the restriction of $\mu$ to $\mathcal{M}_{\mu}$ (considered as a measure defined on a $\sigma$-algebra) is the completion and then saturation (in that order) of $\nu$.

We shall also be interested in two other topological properties of measures; namely $\mathcal{T}$-regular and $\mathcal{T}$-continuous, where $\mathcal{T}$ denotes the family of all open subsets of $X$. A measure $\mu$ is $\mathcal{T}$-regular whenever for all $E \subseteq X$ we have

$$
\mu(E)=\inf \{\mu(T) \mid E \subseteq T \in \mathcal{T}\},
$$

and is $\mathcal{T}$-continuous whenever for all subfamilies $\mathcal{U}$ of $\mathcal{T}$, which are (upwardly) directed by $\subseteq$, we have

$$
\mu(\bigcup \mho)=\sup \{\mu(U) \mid U \in \mathcal{U}\}
$$


All the measures in the discussion that follows will be assumed to be $\mathcal{T}$-continuous, as we wish to relate them to continuous valuations. The condition that $\mu$ be $\mathcal{T}$-regular will only be required in Subsections 4.4 and 7.4. We note that, although all Borel measures are uniquely determined by their restriction to $\mathcal{T}$, the condition of $\mathcal{T}$-regularity gives a simple method by which to reconstruct the original measure from this restriction to $\mathcal{T}$.

The following lemma will be of use to guarantee that a finite $\mathcal{T}$-continuous Borel measure is $\mathcal{T}$-regular. We recall that a topological space $X$ is said to be regular whenever for all points $x \in X$ and closed subsets $F$ of $X$ with $x \notin F$ there exist disjoint open subsets $U$ and $V$ of $X$ such that $x \in U$ and $F \subseteq V$.

Lemma 1. Let $X$ be regular and let $\mu$ be a finite $\mathcal{T}$-continuous Borel measure on $X$. Then $\mu$ is $\mathcal{T}$-regular.

Proof. We consider the family $\mathcal{A}$ of all Borel subsets of $X$ for which $\mathcal{T}$-regularity holds; that is

$$
\mathcal{A}=\{B \in \mathcal{B} \mid \mu(B)=\inf \{\mu(T) \mid B \subseteq T \in \mathcal{T}\}\} .
$$

Since $\mu$ is $\mathcal{B}$-regular, it suffices to show that $\mathcal{A}=\mathcal{B}$. We note that $\mathcal{T} \subseteq \mathcal{A}$ since $\mathcal{T} \subseteq \mathcal{B}$. One readily verifies, using the measurability of all the sets concerned, that $\mathcal{A}$ is closed under finite unions and intersections, and hence also under countable unions and intersections. Thus the result will follow on showing that $\mathcal{A}$ contains the family of all closed subsets of $X$.

Let $F$ be a closed subset of $X$. For each $x \in X \backslash F$ we may, by the regularity of $X$, choose disjoint open subsets $U_{x}$ and $V_{x}$ of $X$ such that $x \in U_{x}$ and $F \subseteq V_{x}$. Letting $\mathcal{U}$ denote the family of all finite unions of the sets $U_{x}$, we see that $X \backslash F=\bigcup \mathcal{U}$. Thus for $\varepsilon>0$ we may choose a finite collection of points, $x(1), \ldots, x(n)$ in $X \backslash F$ such that

$$
\mu\left(\bigcup_{i=1}^{n} U_{x(i)}\right)>\mu(X \backslash F)-\varepsilon
$$

Letting $T=\bigcap_{i=1}^{n} V_{x(i)}$, it follows that $F \subseteq T$, and that

$$
\mu(F) \leqslant \mu(T)<\mu(X)-(\mu(X \backslash F)-\varepsilon)=\mu(F)+\varepsilon,
$$

as required.

\subsection{Normalised continuous valuations on $D$}

To 'construct' the Riemann-Edalat integral on $X$ we will work with the space $\mathrm{P}^{1} D$ of normalised continuous valuations on $D$, which we now define. We define $\mathcal{O}$ to be the family of all Scott open subsets of $D$. A function $v: \mathcal{O} \rightarrow \overline{\mathbb{R}}_{0}^{+}$will be called a valuation on $D$ whenever the following conditions are satisfied:

1. $v(\emptyset)=0$;

2. if $U, V \in \mathcal{O}$ with $U \subseteq V$ then $v(U) \leqslant v(V)$;

3. $v$ is modular; namely for all $U, V \in \mathcal{O}$,

$$
v(U \cup V)+v(U \cap V)=v(U)+v(V) .
$$

A continuous valuation $v$ satisfies the extra condition that for all subfamilies $\mathcal{U}$ of $\mathcal{O}$, which are (upwardly) directed by $\subseteq$, we have

$$
v(\bigcup \mathcal{U})=\sup \{v(U) \mid U \in \mathcal{U}\}
$$


If, in addition, $v(D)=1$ then $v$ is said to be a normalised continuous valuation. We denote the space of all normalised continuous valuations on $D$ by $\mathrm{P}^{1} D$, and define the partial order $\sqsubseteq^{1}$ on $\mathrm{P}^{1} D$ by $v_{1} \sqsubseteq^{1} v_{2}$ whenever $v_{1}(O) \leqslant v_{2}(O)$ for all Scott open sets $O$. As is well known, the partial order $\sqsubseteq^{1}$ induces a way below relation, which we shall denote by $\ll^{1}$; see for example $[3,17]$.

Of special interest are the point valuations $\eta_{d}$ defined for each $d \in D$ by

$$
\eta_{d}(O)= \begin{cases}1 & \text { if } d \in O \\ 0 & \text { if } d \notin O .\end{cases}
$$

A normalised simple valuation is a finite linear combination of point valuations which belongs to $\mathrm{P}^{1} D$; for example

$$
\sum_{a \in A} r_{a} \eta_{a} \quad \text { where } A \subseteq D \text { is finite, and } \sum_{a \in A} r_{a}=1 \text {. }
$$

The embedding $e$ allows us to transfer a Borel measure $\mu$ on $X$ to a valuation $\hat{\mu}$ on $D$ by defining

$$
\hat{\mu}(O)=\mu\left(e^{-1}(O)\right)
$$

for all Scott open subsets $O$; giving a map $\mu \mapsto \hat{\mu}$ from the family of all $\mathcal{T}$-continuous probability Borel measures on $X$ into $\mathrm{P}^{1} D$. We shall use the notation $S(\hat{\mu})$ for the directed set

$$
\left\{v \in \mathrm{P}^{1} D \mid v \text { simple and } v \ll^{1} \hat{\mu}\right\} .
$$

In this way we may think of $S(\hat{\mu})$ as the set of simple valuations approximating $\mu$.

The following two lemmas, taken from [3], will be of use; see also [19, pages 84 and 87].

Lemma 2. For two normalised simple valuations

$$
v_{1}=\sum_{a \in A} r_{a} \eta_{a} \quad \text { and } \quad v_{2}=\sum_{b \in B} s_{b} \eta_{b},
$$

where $A, B \subseteq D$, we have $\nu_{1} \sqsubseteq^{1} \nu_{2}$ if and only if, for all $a \in A$ and $b \in B$, there exists $a$ non-negative number $t_{a, b}$ such that

$$
\sum_{b \in B} t_{a, b}=r_{a} \quad \text { and } \quad \sum_{a \in A} t_{a, b}=s_{b}
$$

and $t_{a, b} \neq 0$ implies that $a \sqsubseteq b$, for all $a \in A$ and $b \in B$.

Lemma 3. For two normalised simple valuations

$$
\nu_{1}=\sum_{a \in A} r_{a} \eta_{a} \quad \text { and } \quad \nu_{2}=\sum_{b \in B} s_{b} \eta_{b},
$$

where $A, B \subseteq D$, we have $v_{1} \ll^{1} v_{2}$ if and only if $\perp \in A$ with $r_{\perp} \neq 0$ and, for all $a \in A$ and $b \in B$, there exists a non-negative number $t_{a, b}$, with $t_{\perp, b} \neq 0$, such that

$$
\sum_{b \in B} t_{a, b}=r_{a} \quad \text { and } \quad \sum_{a \in A} t_{a, b}=s_{b}
$$

and $t_{a, b} \neq 0$ implies that $a \ll b$, for all $a \in A$ and $b \in B$.

We also give a result for $\mathrm{P}^{1} D$ corresponding to [3, Proposition 2.4]; see also [3, Lemma $3.4]$ and [22, page 46]. 
Lemma 4. Let $\lambda$ be a normalised continuous valuation and let $v=\sum_{a \in A} r_{a} \eta_{a}$ be a simple normalised valuation. Then $v \ll^{1} \lambda$ if and only if $\perp \in A$ with $r_{\perp}>0$ and for all non-empty $B \subseteq A \backslash\{\perp\}$ we have

$$
\sum_{b \in B} r_{b}<\lambda(B \uparrow),
$$

where $B \uparrow$ denotes the set $\bigcup_{b \in B} b \uparrow$.

Proof. We will use the notation and methods of [3]. For the 'if' part, note that the conditions above imply by [3, Proposition 2.4] that $v^{-} \ll \lambda$, and hence $v=\left(v^{-}\right)^{+} \ll^{1} \lambda$, by [3, Proposition 3.2(ii)]. For the 'only if' part, first note that by [3, Lemma 3.4] we must have $\perp \in A$ with $r_{\perp}>0$. Therefore, by [3, Proposition 3.2(iii)] we have $v^{-} \ll \lambda$, and the result follows directly from [3, Proposition 2.4].

\section{Definition of the integral}

We first define the integral and then show that it is well defined and linear. Our definition is a straightforward generalization to our framework of that given in [3, Section 4].

\subsection{The construction}

We now define the integral, with respect to a $\mathcal{T}$-continuous probability Borel measure $\mu$, for a bounded function $f: X \rightarrow[\alpha, \beta]$, where $[\alpha, \beta]$ is a (non-empty) interval of $\mathbb{R}$. We define functions $f_{*}: D \rightarrow[\alpha, \beta]$ and $f^{*}: D \rightarrow[\alpha, \beta]$ by

$$
f_{*}(d)=\inf _{x \in[d]} f(x) \text { and } f^{*}(d)=\sup _{x \in[d]} f(x),
$$

with the convention that $\inf \emptyset=\beta$ and $\sup \emptyset=\alpha$. For each $v \in S(\hat{\mu})$ we can express $v$ as $\sum_{a \in A} r_{a} \eta_{a}$, where $A \subseteq D$ and $\sum_{a \in A} r_{a}=1$, and define the Darboux lower sum by

$$
\int f_{*} \mathrm{~d} v=\sum_{a \in A} r_{a} f_{*}(a),
$$

and the Darboux upper sum by

$$
\int f^{*} \mathrm{~d} v=\sum_{a \in A} r_{a} f^{*}(a) .
$$

The Riemann-Edalat lower integral is then defined as

$$
\int_{*} f \mathrm{~d} \mu=\sup _{v \in S(\hat{\mu})} \int f_{*} \mathrm{~d} \nu,
$$

and similarly the Riemann-Edalat upper integral as

$$
\int^{*} f \mathrm{~d} \mu=\inf _{\nu \in S(\hat{\mu})} \int f^{*} \mathrm{~d} \nu .
$$

Finally, when $\int_{*} f \mathrm{~d} \mu=\int^{*} f \mathrm{~d} \mu$ we write $\int f \mathrm{~d} \mu$ for the common value, and say that $f$ is Riemann-Edalat integrable, which we will denote by $f \in \mathcal{R}(\mu)$; that is, $\mathcal{R}(\mu)$ is the family of all bounded functions $f: X \rightarrow \mathbb{R}$ such that $\int f \mathrm{~d} \mu$ exists.

Note 2. We remark that the above construction does not extend directly to unbounded functions (except the special case that $\int f \mathrm{~d} \mu= \pm \infty$ ). For if $v=\sum_{a \in A} r_{a} \eta_{a} \in S(\hat{\mu})$, by 
Lemma 4, we see that $\perp \in A$ with $r_{\perp}>0$. Thus for unbounded $f$ either $\int f^{*} \mathrm{~d} v=\infty$ or $\int f_{*} \mathrm{~d} v=-\infty$ (or both).

\subsection{The integral is well defined}

We will now show that these definitions are sensible. In doing so we will closely follow the steps in [3]; in particular, compare our Lemma 6 and Corollaries 7 and 9 with [3, Proposition 4.2, Corollary 4.3 and Proposition 4.1, respectively]. The proofs of our results, listed above, mimic those given in [3], and are only given for completeness. It may also be advantageous to consider $[\alpha, \beta]$ as an order structure $[\alpha, \beta]_{*}$, ordered by the usual real ordering $\leqslant$, and the dual space $[\alpha, \beta]^{*}$, ordered by the opposite ordering $\leqslant{ }^{\mathrm{OP}}=\geqslant$. Thus $f_{*}: D \rightarrow[\alpha, \beta]_{*}$ and $f^{*}: D \rightarrow[\alpha, \beta]^{*}$ are both order-preserving, but in general may not be continuous.

Lemma 5. Let $v \in S(\hat{\mu})$. Then $\int f_{*} \mathrm{~d} v \leqslant \int f^{*} \mathrm{~d} v$.

Proof. We can express $v$ as

$$
\sum_{a \in A} r_{a} \eta_{a} \quad \text { where } A \subseteq D \text { is finite, and } \sum_{a \in A} r_{a}=1 .
$$

The result follows by noting that for each $a \in A$ we have $f_{*}(a) \leqslant f^{*}(a)$.

Lemma 6. Let $v_{1}, v_{2} \in S(\hat{\mu})$. Suppose $v_{1} \sqsubseteq v_{2}$. Then

$$
\int f_{*} \mathrm{~d} \nu_{1} \leqslant \int f_{*} \mathrm{~d} \nu_{2} \text { and } \int f^{*} \mathrm{~d} \nu_{1} \geqslant \int f^{*} \mathrm{~d} \nu_{2} .
$$

Proof. Express $v_{1}$ as $\sum_{a \in A} r_{a} \eta_{a}$ and $\nu_{2}$ as $\sum_{b \in B} s_{b} \eta_{b}$, where $A, B \subseteq D$ are finite, and the coefficients sum to one. Then by Lemma 2 there exist $t_{a, b} \geqslant 0$ such that for each $a \in A$ and $b \in B$

$$
r_{a}=\sum_{b \in B} t_{a, b} \quad \text { and } \quad s_{b}=\sum_{a \in A} t_{a, b}
$$

and

$$
t_{a, b} \neq 0 \Longrightarrow a \sqsubseteq b .
$$

Thus

$$
\int f_{*} \mathrm{~d} \nu_{1}=\sum_{a \in A}\left(\sum_{b \in B} t_{a, b}\right) f_{*}(a) \leqslant \sum_{b \in B}\left(\sum_{a \in A} t_{a, b}\right) f_{*}(b)=\int f_{*} \mathrm{~d} \nu_{2}
$$

since

$$
t_{a, b} \neq 0 \Longrightarrow a \sqsubseteq b \Longrightarrow[a] \supseteq[b] \Longrightarrow f_{*}(a) \leqslant f_{*}(b) .
$$

A dual argument holds for $f^{*}$.

Corollary 7. Let $v_{1}, v_{2} \in S(\hat{\mu})$. Then $\int f_{*} \mathrm{~d} v_{1} \leqslant \int f^{*} \mathrm{~d} v_{2}$.

Proof. Since $S(\hat{\mu})$ is directed, we can take $v_{3} \in S(\hat{\mu})$ with $v_{1}, v_{2} \sqsubseteq v_{3}$. Then

$$
\int f_{*} \mathrm{~d} \nu_{1} \leqslant \int f_{*} \mathrm{~d} \nu_{3} \leqslant \int f^{*} \mathrm{~d} \nu_{3} \leqslant \int f^{*} \mathrm{~d} \nu_{2} .
$$


Lemma 8. Let $f, g: X \rightarrow \mathbb{R}$ be bounded, $c \in \mathbb{R}_{0}^{+}$and $v \in S(\hat{\mu})$. Then

$$
\begin{gathered}
\int(f+g)_{*} \mathrm{~d} v \geqslant \int f_{*} \mathrm{~d} v+\int g_{*} \mathrm{~d} v \\
\int(f+g)^{*} \mathrm{~d} v \leqslant \int f^{*} \mathrm{~d} v+\int g^{*} \mathrm{~d} v \\
\int(-f)_{*} \mathrm{~d} v=-\int f^{*} \mathrm{~d} v \quad \text { and } \int(-f)^{*} \mathrm{~d} v=-\int f_{*} \mathrm{~d} v, \\
\int(c f)_{*} \mathrm{~d} v=c \int f_{*} \mathrm{~d} v \text { and } \int(c f)^{*} \mathrm{~d} v=c \int f^{*} \mathrm{~d} v,
\end{gathered}
$$

and if $f \leqslant g$ then

$$
\int f_{*} \mathrm{~d} v \leqslant \int g_{*} \mathrm{~d} v \text { and } \int f^{*} \mathrm{~d} v \leqslant \int g^{*} \mathrm{~d} v .
$$

Proof. The result follows easily by noting that for all $a \in D$ we have

$$
(f+g)_{*}(a) \geqslant f_{*}(a)+g_{*}(a), \quad(-f)_{*}=-f^{*} \quad \text { and } \quad(c f)_{*}(a)=c f_{*}(a)
$$

and if $f \leqslant g$ then $f_{*}(a) \leqslant g_{*}(a)$, and similarly for $f^{*}, g^{*}$ with a dual inequality holding for $(f+g)^{*}$.

Corollary 9. The set $\mathcal{R}(\mu)$ of functions, with addition and scalar multiplication defined pointwise, is a real vector space, and integration with respect to $\mu$ is a positive linear functional.

Proof. It follows directly from Lemmas 5 and 8 that

$$
\int^{*} f \mathrm{~d} \mu+\int^{*} g \mathrm{~d} \mu \geqslant \int^{*}(f+g) \mathrm{d} \mu \geqslant \int_{*}(f+g) \mathrm{d} \mu \geqslant \int_{*} f \mathrm{~d} \mu+\int_{*} g \mathrm{~d} \mu,
$$

and hence for $f, g \in \mathcal{R}(\mu)$ we have $f+g \in \mathcal{R}(\mu)$ and

$$
\int(f+g) \mathrm{d} \mu=\int f \mathrm{~d} \mu+\int g \mathrm{~d} \mu
$$

since in this case the left- and right-hand sides of (3.1) must be equal. Similarly, $c f \in \mathcal{R}(\mu)$ and $\int c f \mathrm{~d} \mu=c \int f \mathrm{~d} \mu$, whenever $c \in \mathbb{R}$ and $f \in \mathcal{R}(\mu)$, and $\int f \mathrm{~d} \mu \geqslant 0$ whenever $f \in \mathcal{R}(\mu)$ with $f \geqslant 0$.

\section{Properties of the integral}

The aim of this section is to draw parallels with the classical Riemann integral on compact intervals of $\mathbb{R}$. In particular, we classify all functions $f \in \mathcal{R}(\mu)$ and show that the Lebesgue integral is recaptured by the Bourbaki extension of $\int \cdot \mathrm{d} \mu$ on the lattice of continuous functions on a completely regular, Hausdorff space $X$.

\subsection{A condition for integrability}

Our first result is a restatement of [3, Proposition 4.6], which will prove useful in the remainder of this section. 
Lemma 10 (The $\mathcal{R}$-Condition). Let $f: X \rightarrow \mathbb{R}$ be bounded. Then $f \in \mathcal{R}(\mu)$ if and only if for all $\varepsilon>0$, there exists $v \in S(\hat{\mu})$ such that

$$
\int f^{*} \mathrm{~d} v-\int f_{*} \mathrm{~d} v<\varepsilon
$$

Proof. Let $f \in \mathcal{R}(\mu)$, so that $\int_{*} f \mathrm{~d} \mu=\int^{*} f \mathrm{~d} \mu=\int f \mathrm{~d} \mu$. Let $\varepsilon>0$ and choose $v_{1}, v_{2} \in S(\hat{\mu})$ with

$$
\int f \mathrm{~d} \mu-\int f_{*} \mathrm{~d} \nu_{1}<\frac{\varepsilon}{2} \quad \text { and } \quad \int f^{*} \mathrm{~d} \nu_{2}-\int f \mathrm{~d} \mu<\frac{\varepsilon}{2} .
$$

Since $S(\hat{\mu})$ is directed we may choose $\nu_{3} \in S(\hat{\mu})$ with $v_{1}, \nu_{2} \sqsubseteq \nu_{3}$, so that

$$
\int f^{*} \mathrm{~d} v_{3}-\int f_{*} \mathrm{~d} v_{3}<\varepsilon
$$

Conversely, for $f \notin \mathcal{R}(\mu)$ we may set

$$
\varepsilon=\int^{*} f \mathrm{~d} \mu-\int_{*} f \mathrm{~d} \mu>0 .
$$

Then, for all $v \in S(\hat{\mu})$ we have

$$
\int f^{*} \mathrm{~d} \nu-\int f_{*} \mathrm{~d} \nu \geqslant \int^{*} f \mathrm{~d} \mu-\int_{*} f \mathrm{~d} \mu=\varepsilon,
$$

thus completing the proof.

\subsection{Characterization of integrable functions}

Here we show (see Theorem 12) that an analogue holds for the Riemann-Edalat integral of the well-known Lebesgue criterion which characterizes the Riemann integrable functions on a compact interval of $\mathbb{R}$. Our first task is to find the set of continuity points of a function $f: X \rightarrow \mathbb{R}$.

We define a set $C \subseteq D$ to be a covering of $X$ from $D$ whenever

$$
X \subseteq \bigcup_{c \in C}(c)
$$

and for all $c \in C$ we have $(c) \neq \emptyset$. We let $\mathcal{C}$ denote the family of all coverings of $X$ from $D$ and define the relation $\leqslant$ on $\mathcal{C}$ by $A \leqslant B$ if and only if whenever $x \in X$ and $a \in A$ with $a \ll e(x)$ there is a $b \in B$ with $a \sqsubseteq b \ll e(x)$; that is,

$$
\forall a \in A, \forall x \in X,(a \ll e(x) \Longrightarrow \exists b \in B: a \sqsubseteq b \ll e(x)) .
$$

Observe that if $A \subseteq D$ is such that $X \subseteq \bigcup_{a \in A}(a)$ then

$$
\{a \in A \mid(a) \neq \emptyset\}
$$

is a covering of $X$ from $D$. For $\mathcal{B} \subseteq \mathcal{C}$, a family of coverings of $X$, we say that $\mathcal{B}$ is a fine covering system of $X$ whenever $\mathcal{B}$ is directed with respect to the ordering $\leqslant$, defined above, and

$$
\{(b) \mid \exists B \in \mathcal{B}: b \in B \in \mathcal{B}\}
$$

is a basis of the topology on $X$. An example of such a fine covering system is $\mathcal{C}$. However, in the arguments that follow, it may be useful to consider a smaller family. When $B \subseteq D$ is 


\section{Domain-theoretic integration.}

a basis of approximation in $D$ then we can take

$$
\mathcal{B}=\{A \subseteq B \mid A \text { is a covering of } X \text { from } D\} .
$$

Another example is provided when $X$ is a metric space, $D=\mathcal{J}(\mathbf{B} X)_{\perp}$ and $e=\downarrow \circ \iota$, as discussed in Subsection 2.2. Here it suffices to take any dense set $E \subseteq X$, or $E=X$, and then let

$$
\mathcal{B}=\left\{\left\{\left(x, 2^{-n}\right) \Downarrow \mid x \in E\right\} \mid n \in \mathbb{N}\right\} .
$$

Throughout the rest of this subsection we suppose that a fine covering system $\mathcal{B}$ of $X$ is given.

Let $f: X \rightarrow[\alpha, \beta]$. We define $\Omega_{f}: D \rightarrow[0, \beta-\alpha]$ by

$$
\Omega_{f}(d)=\sup \{f(x)-f(y) \mid x, y \in(d)\} .
$$

For $B \in \mathcal{B}$ and $\varepsilon>0$, we define

$$
G_{B}^{\varepsilon}(f)=\bigcup\left\{(b) \mid b \in B \text { and } \Omega_{f}(b) \leqslant \varepsilon\right\} .
$$

Notice that if $A \leqslant B$ then $G_{A}^{\varepsilon}(f) \subseteq G_{B}^{\varepsilon}(f)$. Hence

$$
G^{\varepsilon}(f)=\bigcup_{B \in \mathcal{B}} G_{B}^{\varepsilon}(f)
$$

is a directed union of open sets. We also note that if $d \in D$ is such that $(d) \cap X \backslash G^{\varepsilon}(f) \neq \emptyset$, then $\Omega_{f}(d)>\varepsilon$, since $\mathcal{B}$ is fine on $X$. Finally,

$$
G(f)=\bigcap_{\varepsilon>0} G^{\varepsilon}(f)=\{x \in X \mid f \text { is continuous at } x\} .
$$

Lemma 11. Let $F \subseteq X$ be closed and let $v=\sum_{a \in A} r_{a} \eta_{a} \in S(\hat{\mu})$. Then

$$
\sum\left\{r_{a} \mid a \in A \text { and }(a) \cap F \neq \emptyset\right\} \geqslant \mu(F) .
$$

Proof. Let

$$
B=\{a \in A \mid(a) \cap F=\emptyset\},
$$

so that

$$
\sum\left\{r_{a} \mid a \in A \text { and }(a) \cap F \neq \emptyset\right\}=\sum_{a \in A \backslash B} r_{a} .
$$

If $B=\emptyset$ then the conclusion holds since $\sum_{a \in A} r_{a}=1 \geqslant \mu(F)$. By Lemma $4, \perp \in A$. If $\perp \in B$ then $F=(\perp) \cap F=\emptyset$, which implies that $B=A$, and the conclusion holds by convention; namely, $\sum \emptyset=0=\mu(\emptyset)$. Thus we may assume that $B \neq \emptyset$ and $B \subseteq A \backslash\{\perp\}$, and hence by Lemma 4 we have

$$
\sum_{b \in B} r_{b}<\hat{\mu}(B \uparrow)=\mu\left(e^{-1}(B \uparrow)\right),
$$

where $B \uparrow$ denotes the set $\bigcup_{b \in B} b \uparrow$. The result follows on noting that for each $b \in B$ we have $(b) \subseteq X \backslash F$, so that $e^{-1}(B \uparrow)=\bigcup_{b \in B}(b) \subseteq X \backslash F$, and consequently

$$
\sum_{a \in A \backslash B} r_{a}>1-\mu\left(e^{-1}(B \uparrow)\right) \geqslant 1-\mu(X \backslash F)=\mu(F)
$$

as required. 
We can now show our main theorem, which extends [3, Theorem 6.5] and [12, Theorem 5.12] to the framework of this paper.

Theorem 12. Let $f: X \rightarrow[\alpha, \beta]$ be bounded. Then $f \in \mathcal{R}(\mu)$ if and only if $f$ is continuous at $\mu$-almost all $x \in X$.

Proof. Suppose that $f$ is not continuous at $\mu$-almost all $x \in X$; that is $\mu(G(f))<1$. Then for some $\varepsilon>0$ we have $\mu\left(G^{\varepsilon}(f)\right)<1$, and hence the closed set $X \backslash G^{\varepsilon}(f)$ has positive measure. Thus for all $v=\sum_{a \in A} r_{a} \eta_{a} \in S(\hat{\mu})$ we have

$$
\int f^{*} \mathrm{~d} v-\int f_{*} \mathrm{~d} v=\sum_{a \in A} r_{a} \sup _{x, y \in[a]}(f(x)-f(y)) \geqslant \varepsilon \mu\left(X \backslash G^{\varepsilon}(f)\right)>0,
$$

since if $a \in A$ is such that $(a) \cap\left(X \backslash G^{\varepsilon}(f)\right) \neq \emptyset$ then

$$
\sup _{x, y \in[a]}(f(x)-f(y))>\varepsilon .
$$

Therefore, the $\mathcal{R}$-condition fails.

The converse will be shown by checking that the $\mathcal{R}$-condition holds. Suppose that $f$ is continuous $\mu$-almost everywhere, and let $\varepsilon>0$. Then $\mu\left(G^{\varepsilon}(f)\right)=1$. Hence we may take $B \in \mathcal{B}$ with $\mu\left(G_{B}^{\varepsilon}(f)\right)>1-\varepsilon$. We define

$$
O=\bigcup\left\{b \uparrow \mid b \in B \text { and } \Omega_{f}(b) \leqslant \varepsilon\right\}
$$

so that $e^{-1}(O)=G_{B}^{\varepsilon}(f)$. Thus $\hat{\mu}(O)=\mu\left(G_{B}^{\varepsilon}(f)\right)>1-\varepsilon$, and hence we may take $v=\sum_{a \in A} r_{a} \eta_{a} \in S(\hat{\mu})$ with $v(O)>1-\varepsilon$. Thus $\sum_{a \in A \backslash O} r_{a}<\varepsilon$ and hence

$$
\sum_{a \in A \backslash O} r_{a}\left(f^{*}(a)-f_{*}(a)\right)<(\beta-\alpha) \varepsilon .
$$

For each $a \in A \cap O$ there exists $b \in B$ such that

$$
b \ll a \quad \text { and } \quad \Omega_{f}(b) \leqslant \varepsilon .
$$

Thus

$$
\sup _{x \in[a]} f(x)-\inf _{y \in[a]} f(y)=f^{*}(a)-f_{*}(a) \leqslant \varepsilon,
$$

and hence $\sum_{a \in A \cap O} r_{a}\left(f^{*}(a)-f_{*}(a)\right) \leqslant \varepsilon$. Therefore,

$$
\int f^{*} \mathrm{~d} v-\int f_{*} \mathrm{~d} v=\sum_{a \in A} r_{a}\left(f^{*}(a)-f_{*}(a)\right)<\varepsilon+(\beta-\alpha) \varepsilon=(1+\beta-\alpha) \varepsilon,
$$

and the result follows.

\subsection{A connection with Lebesgue integration}

In this subsection we show that if a function is Riemann-Edalat integrable then it is Lebesgue summable, and the values of the integrals agree. This extends [3, Theorem 7.2] and [12, Theorem 5.13] to our setting; our proof is similar to that of [12, Theorem 5.13]. There are a number of (equivalent) definitions of the Lebesgue integral of a function $f: X \rightarrow \overline{\mathbb{R}}$ with respect to a measure $\mu$, which we shall denote here by $\mu(f)$. We give one such definition which will suit our purposes; see, for example, [16, Section 2.4]. 
We let $\mathcal{M}$ denote the family of all $\mu$-measurable subsets of $X$, and let $u: X \rightarrow \overline{\mathbb{R}}$. We recall that $u$ is said to be a $\mu$-measurable function whenever

$$
\{x \in X \mid u(x)>t\} \in \mathcal{M}
$$

for all $t \in \mathbb{R}$. We say that $u: X \rightarrow \overline{\mathbb{R}}$ is a $\mu$-step function if and only if $u$ is a $\mu$-measurable function with countable image $u(X) \subseteq \overline{\mathbb{R}}$ such that

$$
\sum_{t \in u(X)} t \mu\left(u^{-1}(t)\right)=\sum_{t \in u^{+}(X)} t \mu\left(u^{-1}(t)\right)-\sum_{t \in u^{-}(X)} t \mu\left(u^{-1}(t)\right) \in \overline{\mathbb{R}} ;
$$

that is, the right-hand side of the equality is not of the form $\infty-\infty$. The usual convention of $\pm \infty \cdot 0=0=0 \cdot \pm \infty$ is employed here.

Let $f: X \rightarrow \overline{\mathbb{R}}$ be any function. The upper Lebesgue integral of $f$ with respect to $\mu$ is defined by

$$
\mu^{*}(f)=\inf \left\{\sum_{t \in u(X)} t \mu\left(u^{-1}(t)\right) \mid f \leqslant u \text { is a } \mu \text {-step function }\right\},
$$

and similarly the lower Lebesgue integral is defined by

$$
\mu_{*}(f)=\sup \left\{\sum_{t \in u(X)} t \mu\left(u^{-1}(t)\right) \mid f \geqslant u \text { is a } \mu \text {-step function }\right\} .
$$

We say that $f$ is Lebesgue $\mu$-integrable whenever $f$ is a $\mu$-measurable function and $\mu^{*}(f)=$ $\mu_{*}(f)$, and we define $\mu(f)$ to be the common value. By a Lebesgue $\mu$-summable function $f$ we mean a Lebesgue $\mu$-integrable function $f$ such that $\mu(f) \neq \pm \infty$. The family of all Lebesgue $\mu$-summable functions $f: X \rightarrow \overline{\mathbb{R}}$ will be denoted by $\mathcal{L}(\mu)$. We note that if $\mu^{*}(f)=\mu_{*}(f) \neq \pm \infty$ then $f$ is $\mu$-measurable, and hence $f \in \mathcal{L}(\mu)$. We also note that if $u$ is a $\mu$-step function then $u$ is Lebesgue $\mu$-integrable and

$$
\mu(u)=\sum_{t \in u(X)} t \mu\left(u^{-1}(t)\right)
$$

Theorem 13. Let $f \in \mathcal{R}(\mu)$. Then $f \in \mathcal{L}(\mu)$ and $\int f \mathrm{~d} \mu=\mu(f)$.

Proof. Choose a sequence of simple valuations $\left(v_{i} \in S(\hat{\mu})\right)_{i=1}^{\infty}$ with

$$
\int f^{*} \mathrm{~d} \nu_{i} \rightarrow \int f \mathrm{~d} \mu \text { and } \int f_{*} \mathrm{~d} \nu_{i} \rightarrow \int f \mathrm{~d} \mu \quad \text { as } i \rightarrow \infty .
$$

We write $v_{i}=\sum_{a \in A_{i}} r_{a, i} \eta_{a}$ and define

$$
\begin{aligned}
& u_{i}(x)=\min \left\{f^{*}(a) \mid a \in A_{i} \text { and } x \in(a)\right\}, \\
& v_{i}(x)=\max \left\{f_{*}(a) \mid a \in A_{i} \text { and } x \in(a)\right\},
\end{aligned}
$$

so that $u_{i} \geqslant f \geqslant v_{i}$. We work with $v_{i}$ for the time being.

We order the values of $f_{*}(a)$ for $a \in A_{i}$ as $c_{0}<\cdots<c_{n}$ and define for $j=0, \ldots, n$

$$
B_{i}(j)=\left\{a \in A_{i} \mid f_{*}(a) \geqslant c_{j}\right\} \quad \text { and } \quad U_{i}(j)=e^{-1}\left(B_{i}(j) \uparrow\right)=\bigcup_{b \in B_{i}(j)}(b) .
$$


Note by Lemma 4 that $\perp \in A_{i}$ and hence $c_{0}=f_{*}(\perp)$ and $B_{i}(0)=A_{i}$. We also note that for $j=1, \ldots, n$ we have $\emptyset \neq B_{i}(j) \subseteq A_{i} \backslash\{\perp\}$ and $B_{i}(j) \subset B_{i}(j-1)$. Next we write $\tilde{c}_{0}=c_{0}$ and $\tilde{c}_{j}=c_{j}-c_{j-1}$ for $j=1, \ldots, n$. Thus

$$
v_{i}=\sum_{j=0}^{n} \tilde{c}_{j} \mathbb{1}_{U_{i}(j)}
$$

and hence $v_{i}$ is a $\mu$-step function. Also

$$
\mu\left(v_{i}\right)=\sum_{j=0}^{n} \tilde{c}_{j} \mu\left(U_{i}(j)\right) \geqslant \sum_{j=0}^{n} \tilde{c}_{j}\left(\sum_{b \in B_{i}(j)} r_{b, i}\right)=\sum_{a \in A_{i}} r_{a, i} f_{*}(a)=\int f_{*} \mathrm{~d} v_{i} .
$$

Dually, ordering the values of $f^{*}(a)$ for $a \in A_{i}$ as $d_{0}>\cdots>d_{m}$ we may represent

$$
u_{i}=\sum_{j=0}^{m} \tilde{d}_{j} \mathbb{1}_{V_{i}(j)}
$$

where $\tilde{d}_{j}<0$ for $j=1, \ldots, m$ and show that

$$
\mu\left(u_{i}\right) \leqslant \sum_{a \in A_{i}} r_{a, i} f^{*}(a)=\int f^{*} \mathrm{~d} v_{i}
$$

Thus for $i=1,2, \ldots$ we see that $u_{i}$ and $v_{i}$ are $\mu$-step functions and

$$
\int f^{*} \mathrm{~d} v_{i} \geqslant \mu\left(u_{i}\right) \geqslant \mu^{*}(f) \geqslant \mu_{*}(f) \geqslant \mu\left(v_{i}\right) \geqslant \int f_{*} \mathrm{~d} v_{i} .
$$

Letting $i \rightarrow \infty$ we see that

$$
\mu^{*}(f)=\mu_{*}(f)=\int f \mathrm{~d} \mu \neq \pm \infty
$$

and the result follows.

\subsection{Bourbaki extensions of the integral}

We begin this subsection by defining the notion of a Bourbaki integral on a Stonian lattice of functions. Our treatment (and terminology) is in the spirit of [16, Section 2.5], except that we use Bourbaki extensions instead of Daniell extensions. The text [26] gives an excellent account of both the Bourbaki and Daniell extensions of an integral defined on a vector lattice of functions (defined in [26, 2.1], and called a fundamental system). Here our first extension of the integral is from the Stonian lattice $\mathcal{S}$ to the vector lattice $\mathcal{S}-\mathcal{S}$, see below. All subsequent steps are as in [26], and thus our treatment here is essentially equivalent to that of [26].

A family $\mathcal{S}$ of functions $f: X \rightarrow \mathbb{R}$ is a Stonian lattice if and only if whenever $c \in \mathbb{R}_{0}^{+}$ and $f, g \in \mathcal{S}$ then

$$
f+g, \quad c f, \quad f \wedge g, \quad f \wedge c
$$

are all in $\mathcal{S}$, and if $f \leqslant g$ then $g-f \in \mathcal{S}$.

Observe that $f^{+}=f-f \wedge 0, f^{-}=f^{+}-f$ and $|f|=f^{+}+f^{-}$are in $\mathcal{S}$. Also, if $\mathcal{S}$ is a Stonian lattice, then so is

$$
\mathcal{S}^{+}=\{f \in \mathcal{S} \mid f \geqslant 0\}
$$


Finally, the main example that we wish to consider is the case where $\mathcal{S}$ is the family of all bounded continuous functions $f: X \rightarrow \mathbb{R}$.

A function $I: S \rightarrow \mathbb{R}$ is a positive Bourbaki integral (on the Stonian lattice $\mathcal{S}$ ) means that whenever $f, g \in \mathcal{S}, c \in \mathbb{R}_{0}^{+}$and $\mathcal{F} \subseteq \mathcal{S}$ is downwardly directed with $\mathcal{F} \searrow 0$, that is $\inf _{f \in \mathcal{F}} f(x)=0$ for all $x \in X$, we have

$$
I(f+g)=I(f)+I(g), \quad I(c f)=c I(f), \quad \inf _{f \in \mathcal{F}} I(f)=0,
$$

and if $f \leqslant g$ then $I(f) \leqslant I(g)$.

We note that the following proposition and Corollary 9 imply that $I(f)=\int f \mathrm{~d} \mu$ is a positive Bourbaki integral on the Stonian lattice $\mathcal{S}$ of bounded continuous functions on $X$, and so also on $\mathcal{S}^{+}$.

Proposition 14. Let $\mathcal{F}$ be a downwardly directed family of bounded, continuous, nonnegative functions with $\mathcal{F} \searrow 0$. Then

$$
\inf _{f \in \mathcal{F}} \int f \mathrm{~d} \mu=0 .
$$

Proof. Without loss of generality we may suppose that $\beta$ may be chosen so that $f: X \rightarrow$ $[0, \beta]$ for all $f \in \mathcal{F}$. If there were no such $\beta$, then replacing $\mathcal{F}$ by the (downwardly directed) subfamily $\mathcal{G}=\{f \in F \mid f \leqslant g\}$ in the following argument, for some chosen $g \in \mathcal{F}$ (which by definition is bounded), shows that

$$
\inf _{f \in \mathcal{G}} \int f \mathrm{~d} \mu=0 .
$$

The result then follows on noting that

$$
0 \leqslant \inf _{f \in \mathcal{F}} \int f \mathrm{~d} \mu \leqslant \inf _{f \in \mathcal{G}} \int f \mathrm{~d} \mu,
$$

since $\mathcal{F}$ must be non-empty and every $f \in \mathcal{F}$ is non-negative.

Let $\varepsilon>0$ and define for each $f \in \mathcal{F}$

$$
G^{\varepsilon}(f)=f^{-1}([0, \varepsilon)),
$$

so that $X=\bigcup_{f \in \mathcal{F}} G^{\varepsilon}(f)$ is a directed union of open sets. Thus there exists $f \in \mathcal{F}$ such that $\mu\left(G^{\varepsilon}(f)\right)>1-\varepsilon$, since $\mu(X)=1$. Define

$$
O=\bigcup\left\{d \uparrow \mid d \in D \text { and }(d) \subseteq G^{\varepsilon}(f)\right\}
$$

so that $e^{-1}(O)=G^{\varepsilon}(f)$ and hence $\hat{\mu}(O)>1-\varepsilon$. Choose $v=\sum_{a \in A} r_{a} \eta_{a} \in S(\hat{\mu})$ with $\nu(O)>1-\varepsilon$. Then

$$
\sum_{a \in A \backslash O} r_{a} f^{*}(a)<\varepsilon \beta
$$

and

$$
\sum_{a \in A \cap O} r_{a} f^{*}(a) \leqslant \sum_{a \in A \cap O} r_{a} \varepsilon \leqslant \varepsilon,
$$

since if $a \in O$ then there is a $d \in D$ with $d \ll a$ and $[a] \subseteq(d) \subseteq G^{\varepsilon}(f)$, so that $f^{*}(a) \leqslant \varepsilon$. Therefore,

$$
0 \leqslant \int f_{*} \mathrm{~d} \nu \leqslant \int f^{*} \mathrm{~d} \nu<\varepsilon+\varepsilon \beta=(1+\beta) \varepsilon,
$$


and hence

$$
0 \leqslant \int f \mathrm{~d} \mu \leqslant(1+\beta) \varepsilon .
$$

Since $\varepsilon$ was arbitrary, the result follows.

We recall that the topological space $X$ is said to be completely regular whenever for all points $x \in X$ and $U \subseteq X$ open with $x \in U$ there exists a continuous function $f: X \rightarrow[0,1]$ such that $f(x)=1$ and $f(y)=0$ for all $y \in X \backslash U$. We also note that if $X$ is completely regular then it is also regular. In this case, the following theorem allows us to recapture the measure $\mu$ from the Bourbaki integral $I(f)=\int f \mathrm{~d} \mu$ defined on the lattice of bounded continuous functions on $X$. For an interesting example of what can go wrong if we were to use the Daniell theory, see [16, 2.5.15].

We use the notation $\mathcal{G}_{\tau}$, where $\mathcal{G}$ is a family of subsets of $X$, to denote the closure of $\mathcal{G}$ under directed unions; that is,

$$
\mathcal{G}_{\tau}=\{\bigcup \mathcal{F} \mid \mathcal{F} \subseteq \mathcal{G} \text { and } \mathcal{F} \text { is directed under } \subseteq\} .
$$

We shall also use the following terminology. A measure $\phi$ on $X$ is a $\mathcal{G}_{\tau}$-measure whenever $\mathcal{G}_{\tau} \subseteq \mathcal{M}_{\phi}$ (that is, each set in $\mathcal{G}_{\tau}$ is $\phi$-measurable) and $\phi$ is $\mathcal{G}_{\tau}$-regular; that is, for all $E \subseteq X$

$$
\phi(E)=\inf \left\{\phi(G) \mid E \subseteq G \in \mathcal{G}_{\tau}\right\}
$$

with the convention that $\inf \emptyset=\infty$. We will say that $\phi$ is a continuous $\mathcal{G}_{\tau}$-measure if $\phi$ is a $\mathcal{G}_{\tau}$-measure and $\phi$ is $\mathcal{G}_{\tau}$-continuous; that is, for all subfamilies $\mathcal{H}$ of $\mathcal{G}_{\tau}$, which are (upwardly) directed by $\subseteq$, we have

$$
\phi(\bigcup \mathcal{H})=\sup \{\phi(H) \mid H \in \mathcal{H}\} .
$$

Theorem 15. Let $\mathcal{S}$ be a Stonian lattice of functions on $X$, and let I be a positive Bourbaki integral on $\mathcal{S}$. Define

$$
\mathcal{G}=\left\{\{x \in X \mid f(x)>t\} \mid f \in \mathcal{S}^{+} \text {and } t>0\right\} .
$$

Then there exists a unique continuous $\mathcal{G}_{\tau}$-measure $\phi$ on $X$ such that for all $f \in \mathcal{S}$ we have

$$
I(f)=\phi(f),
$$

where $\phi(f)$ denotes the Lebesgue integral of $f$ with respect to $\phi$.

Proof. The proof of this is similar to the proof of the Daniell-Stone Theorem as in [16, 2.5.2 and 2.5.3], except that we need to replace increasing sequences of functions by upwardly directed families $\mathcal{F}$. The measure $\phi$ is defined as follows. For $E \subseteq X$, let us say that $\mathcal{F} \subseteq \mathcal{S}^{+}$ suits $E$ if and only if $\mathcal{F}$ is upwardly directed and $\sup \mathcal{F} \geqslant \mathbb{1}_{E}$; that is, $\sup _{f \in \mathcal{F}} f(x) \geqslant 1$ whenever $x \in E$. Then we define

$$
\phi(E)=\inf \left\{\sup _{f \in \mathcal{F}} I(f) \mid \mathcal{F} \subseteq \mathcal{S}^{+} \text {and } \mathcal{F} \text { suits } E\right\} .
$$

Notice that if $\mathcal{F} \subseteq \mathcal{S}$ is upwardly directed and $\sup \mathcal{F} \geqslant \mathbb{1}_{E}$ then $\left\{f^{+} \mid f \in \mathcal{F}\right\} \subseteq \mathcal{S}^{+}$ suits $E$ and $\sup _{f \in \mathcal{F}} I\left(f^{+}\right)=\sup _{f \in \mathcal{F}} I(f)$, since by the continuity of $I$ on $\mathcal{S}$ we have $\inf _{f \in \mathcal{F}} I\left(f^{-}\right)=0$.

The proofs that

1. $\phi$ is a measure on $X$, 
2. if $E \subseteq X$ and $g \in \mathcal{S}^{+}$with $g \leqslant \mathbb{1}_{E}$ then $I(g) \leqslant \phi(E)$,

3. every member $f \in \mathcal{S}^{+}$is a $\phi$-measurable function,

may be transcribed from the proof of [16, Theorem 2.5.2] by replacing increasing sequences of functions by the appropriate directed subfamilies of $\mathcal{S}^{+}$. Also, the proof that $I(f)=\phi(f)$ for all $f \in \mathcal{S}$ is precisely as in [16, Theorem 2.5.2].

Then, as noted in $[\mathbf{1 6}, 2.5 .3]$,

$$
(f \wedge(t+r)-f \wedge t) / r \nearrow \mathbb{1}_{\{x \in X \mid f(x)>t\}} \quad \text { as } r \searrow 0
$$

whenever $f \in \mathcal{S}^{+}$and $t>0$. It follows that $H \in \mathcal{G}_{\tau}$ if and only if there exists a directed family $\mathcal{H} \subseteq \mathcal{S}^{+}$such that $\mathbb{1}_{H}=\bigvee \mathcal{H}$. We need to show that each member of $\mathcal{G}_{\tau}$ is $\phi$-measurable, and $\phi$ is $\mathcal{G}_{\tau}$-regular and $\mathcal{G}_{\tau}$-continuous.

Let $H \in \mathcal{G}_{\tau}$ and $\mathcal{H} \subseteq \mathcal{S}^{+}$be such that $\mathbb{1}_{H}=\bigvee \mathcal{H}$. Then, in order to prove measurability, we show that for $E \subseteq X$ we have

$$
\phi(E) \geqslant \phi(E \cap H)+\phi(E \backslash H),
$$

as the reverse inequality follows by the definition that $\phi$ is a measure. Thus we may assume that $\phi(E)<\infty$. Let $\mathcal{F} \subseteq \mathcal{S}^{+}$suit $E$ and suppose that $\sup _{f \in \mathcal{F}} I(f)<\infty$. We define $\mathcal{K}=\{f \wedge h \mid f \in \mathcal{F}$ and $h \in \mathcal{H}\}$ and for each $h \in \mathcal{H}$ we define $\mathcal{F}(h)=\{f-f \wedge h \mid f \in \mathcal{F}\}$. Then $\mathcal{K}$ suits $E \cap H$, and hence for all $\varepsilon>0$ we can choose $g \in \mathcal{F}$ and $h \in \mathcal{H}$ such that

$$
I(g \wedge h)>\sup _{k \in \mathcal{K}} I(k)-\varepsilon
$$

since $\sup _{k \in \mathcal{K}} I(k) \leqslant \sup _{f \in \mathcal{F}} I(f)$, which is finite. It follows that $\mathcal{F}(h)$ suits $E \backslash H$, since $f \wedge h \leqslant \mathbb{1}_{H}$ and that

$$
\phi(E \cap H)+\phi(E \backslash H) \leqslant I(g \wedge h)+\varepsilon+\sup _{f \in \mathcal{F}(h)} I(f) \leqslant \sup _{f \in \mathcal{F}} I(f)+\varepsilon .
$$

The inequality (4.2) follows on letting $\varepsilon$ tend to zero and taking the infimum over all families $\mathcal{F}$ that suit $E$.

The fact that $\phi$ is $\mathcal{G}_{\tau}$-continuous follows easily by standard techniques from equation (4.1) and the fact that $I$ is continuous on $\mathcal{S}^{+}$. To prove $\mathcal{G}_{\tau}$-regularity, we let $E \subseteq X$ be such that $\phi(E)<\infty$, and let $\varepsilon>0$. Then we may choose $\mathcal{F} \subseteq \mathcal{S}^{+}$such that $\mathcal{F}$ suits $E$ and $\sup _{f \in \mathcal{F}} I(f)<\phi(E)+\varepsilon$. Then

$$
G=\bigcup_{f \in \mathcal{F}}\{x \in X \mid f(x)>1 /(1+\varepsilon)\}
$$

satisfies $E \subseteq G$ and

$$
\phi(G) \leqslant(1+\varepsilon) \sup _{f \in \mathcal{F}} I(f)<(1+\varepsilon)(\phi(E)+\varepsilon) .
$$

Regularity follows on letting $\varepsilon \searrow 0$.

Finally, for a measure $\theta$ such that all functions $f \in \mathcal{S}^{+}$are $\theta$-measurable and $\theta(f)=$ $I(f)$, we see by equation (4.1) that $\theta$ must agree on $\mathcal{G}$ with $\phi$. It follows that if $\theta$ is a continuous $\mathcal{G}_{\tau}$-measure then $\theta=\phi$.

Notice that when $X$ is completely regular and $S$ is the Stonian lattice of bounded continuous functions on $X$, then $\mathcal{G}$, above, is a family of open subsets of $X$ which is a basis of the topology on $X$ and is closed under finite unions. Hence $\mathcal{G}_{\tau}$ is the family $\mathcal{T}$ of all open subsets of $X$. Thus the unique measure $\phi$ is precisely the original measure $\mu$, by Lemma 1 
and Theorems 12 and 13. Furthermore, it is then the case that $\overline{\mathbf{I}}$, the Bourbaki extension of $I(f)=\int f \mathrm{~d} \mu$, recaptures the Lebesgue integral with respect to $\mu=\phi$; that is, for all $f: X \rightarrow \overline{\mathbf{R}}$ we have $\overline{\mathbf{I}}(f)=\mu(f)$ whenever either side is defined. This is proved in [26, Theorem 12.18 and Corollary 12.22]; see also [26, Remark 10.2].

We briefly sketch the steps in the Bourbaki extension $\overline{\mathbf{I}}$ of a general Bourbaki integral $I$ defined on a Stonian lattice $\mathcal{S}$. We let $\mathcal{S}-\mathcal{S}$ denote the family of functions

$$
\{f-g \mid f, g \in \mathcal{S}\}=\left\{f-g \mid f, g \in \mathcal{S}^{+}\right\} .
$$

Notice that if $\mathcal{S}$ is a vector lattice then $\mathcal{S}-\mathcal{S}=\mathcal{S}$. We extend (if necessary) $I$ to $\mathcal{S}-\mathcal{S}$ by defining $I(f-g)=I(f)-I(g)$ which uniquely determines the value of $I(f-g)$ by the linearity of $I$ and the order properties of $\mathcal{S}$.

Next we let $(\mathcal{S}-\mathcal{S})^{*}$ denote the family of all functions $g$ such that there is an upwardly directed family $\mathcal{F} \subseteq \mathcal{S}-\mathcal{S}$ with $\mathcal{F} \nearrow g$ and define $I^{*}:(\mathcal{S}-\mathcal{S})^{*} \rightarrow \overline{\mathbb{R}}$ by setting

$$
I^{*}(g)=\sup _{f \in \mathcal{F}} I(f)
$$

where $\mathcal{F}$ and $g$ are as above. The value of $I^{*}(g)$ is uniquely determined by the continuity of $I$ on $\mathcal{S}$. Dually, we define $(\mathcal{S}-\mathcal{S})_{*}$ and $I_{*}$ by replacing 'upwardly directed' by 'downwardly directed'. For all functions $f: X \rightarrow \overline{\mathbb{R}}$ we define the upper integral $I^{\#}$ by

$$
I^{\#}(f)=\inf \left\{I^{*}(g) \mid g \in(\mathcal{S}-\mathcal{S})^{*} \text { and } g \geqslant f\right\}
$$

and similarly the lower integral $I_{\#}$ by

$$
I_{\#}(f)=\sup \left\{I_{*}(g) \mid g \in(\mathcal{S}-\mathcal{S})_{*} \text { and } g \leqslant f\right\}
$$

with the convention that $\inf \emptyset=\infty$ and $\sup \emptyset=-\infty$. In the case when $\mathcal{S}-\mathcal{S}=\mathcal{S}$ we simply write $\mathcal{S}^{*}$ for $(\mathcal{S}-\mathcal{S})^{*}$ and $\mathcal{S}_{*}$ for $(\mathcal{S}-\mathcal{S})_{*}$ in the above definitions.

A function $f: X \rightarrow \overline{\mathbb{R}}$ is said to be $I$-summable whenever $I^{\#}(f)=I_{\#}(f) \neq \pm \infty$, and in this case we denote the common value by $\mathbf{I}(f)$. The family of all $I$-summable functions is denoted by $\mathcal{L}(I)$. For the last extension, we let $\mathcal{L}_{+}(I)$ denote the family of all functions $f: X \rightarrow \overline{\mathbb{R}}$ such that $f \wedge g \in \mathcal{L}(I)$ for all $g \in \mathcal{S}-\mathcal{S}$, and dually_ $\mathcal{L}_{-}(I)$ contains all functions such that $f \vee g \in \mathcal{L}(I)$ for all $g \in \mathcal{S}-\mathcal{S}$. Finally, we let $\overline{\mathcal{L}}(I)=\mathcal{L}_{+}(I) \cup \mathcal{L}_{-}(I)$ and extend $\mathbf{I}$ to $\overline{\mathbf{I}}: \overline{\mathcal{L}}(I) \rightarrow \overline{\mathbb{R}}$ by setting $\overline{\mathbf{I}}(f)=I^{\#}(f)$ whenever $f \in \mathcal{L}_{+}(I)$ and $\overline{\mathbf{I}}(f)=I_{\#}(f)$ whenever $f \in \mathcal{L}_{-}(I)$. This is well defined since $\mathcal{L}(I)=\mathcal{L}_{+}(I) \cap \mathcal{L}_{-}(I)$; see for example [26, Remark 6.6]. The functions in $\overline{\mathcal{L}}(I)$ are said to be $I$-integrable.

A full account of this procedure is given in [26]. We also remark that the measure $\phi$, in Theorem 15, may simply be defined for $E \subseteq X$ by $\phi(E)=I^{\#}\left(\mathbb{1}_{E}\right)$, and that $E$ is $\phi$-measurable if and only if $\mathbb{1}_{E} \in \mathcal{L}_{+}(I)$.

\section{Integration on D-Suslin subsets}

In this section we suppose that $A$ is a non-empty subset of $X$ of a certain form; namely, a $D$-Suslin set. A $\mathcal{T}$-continuous probability Borel measure $\mu$ is said to be supported on $A$ whenever $\mu(X \backslash A)=0$. In this case the $D$-Suslin structure of $A$, together with enough knowledge of $\mu$, directly leads to a construction of a sequence $\left(v_{n}\right)_{n=0}^{\infty}$ of simple valuations that approximates $\mu$ with $\hat{\mu}=\bigsqcup_{n=0}^{\infty} v_{n}$ and hence the integral of a function on $X$ may be approximated by evaluating the Darboux sums for this sequence. The next section will deal with identifying which sets are $D$-Suslin. We also note that for the weakly hyperbolic iterated function systems with probabilities, as discussed in [6], the attractor of the IFS is 
$D$-Suslin and the invariant measure is a $\mathcal{T}$-continuous probability Borel measure supported on the attractor; see also [5, Section 6] and [10, Section 6].

Another motivation for this section is to define the integral over a ( $D$-Suslin) set $A$ with respect to a $\mathcal{T}$-continuous Borel measure $\mu$ on $X$ for which $0<\mu(A)<\infty$; we do not assume that $\mu$ is supported on $A$. This may be achieved in the following way. We define the measure $\mu / A$ by

$$
(\mu / A)(E)=\frac{\mu(E \cap A)}{\mu(A)}
$$

for all $E \subseteq X$. Then for any $f: X \rightarrow \mathbb{R}$ we may define

$$
\int_{A} f \mathrm{~d} \mu=\mu(A) \int f \mathrm{~d}(\mu / A)
$$

whenever the right-hand side exists. We show in Subsection 5.5 that $\mu / A$ is a $\mathcal{T}$-continuous probability Borel measure whenever $A$ is a $D$-Suslin set, and consequently the right-hand side of this definition may be evaluated by a sequence of simple valuations.

\subsection{The Suslin operation}

We start with the usual notations to define the Suslin operation. For $n \in \mathbb{N}$, as usual $\mathbb{N}^{n}$ consists of all sequences of natural numbers $\kappa=\left(\kappa_{1}, \ldots, \kappa_{n}\right)$, interpreting $\mathbb{N}^{0}$ by $\{()\}$; the set of the empty sequence ( ). Then $\mathbb{N}^{\infty}$ denotes the disjoint union of the $\mathbb{N}^{n}$ over all $n \in \mathbb{N}$. At last, $\mathbb{N}^{\mathbb{N}}$ is defined to consist of all sequences $\mathbf{i}=\left(i_{\lambda} \in \mathbb{N}\right)_{\lambda=1}^{\infty}$. For $\kappa=\left(\kappa_{1}, \ldots, \kappa_{n}\right) \in \mathbb{N}^{n}$ and $m \in \mathbb{N}$ with $m \leqslant n$ we write $\kappa \mid m=\left(\kappa_{1}, \ldots, \kappa_{m}\right) \in \overline{\mathbb{N}}^{m}$; we do not define $\kappa \mid m$ for $m>n$. Similarly, when $\mathbf{i} \in \mathbb{N}^{\mathbb{N}}$ we write $\mathbf{i} \mid n=\left(i_{1}, \ldots, i_{n}\right) \in \mathbb{N}^{n}$ for all $n \in \mathbb{N}$. Finally, again when $\kappa=\left(\kappa_{1}, \ldots, \kappa_{n}\right) \in \mathbb{N}^{n}$ and $i \in \mathbb{N}$ we will write $\kappa \cdot i=\left(\kappa_{1}, \ldots, \kappa_{n}, i\right) \in \mathbb{N}^{n+1}$, for the concatenation of (the finite sequence) $\kappa$ followed by the sequence $(i)$.

Next, let $\mathcal{F}$ be any class of subsets of $X$; then the Suslin-F sets are the sets of the form

$$
S=\bigwedge_{\kappa \in \mathbb{N}^{\infty}} F(\kappa)=\bigcup_{\mathbf{i} \in \mathbb{N}^{\mathbb{N}}} \bigcap_{n=0}^{\infty} F(\mathbf{i} \mid n)
$$

where $(F(\kappa) \in \mathcal{F})_{\kappa \in \mathbb{N} \infty}$ is a subfamily of $\mathcal{F}$, or equivalently $F: \mathbb{N}^{\infty} \rightarrow \mathcal{F}$. Of special interest (see Section 6) is the case when $\mathcal{F}$ is the family of all closed subsets of $X$. We may refer to $S$ as the set resulting from the Suslin operation applied to $F: \mathbb{N}^{\infty} \rightarrow \mathcal{F}$. Alternatively, we will simply say that $F$ generates $S$.

As is well known, see for example $[16,20,23,27]$, the family of all Suslin- $\mathcal{F}$ sets, where $\mathcal{F}$ is any family of sets, contains $\mathcal{F}$ and is closed under countable unions, countable intersections and the Suslin operation; that is, $\mathfrak{S}(\mathfrak{S}(\mathcal{F}))=\mathfrak{S}(\mathcal{F})$, where $\mathfrak{S}(\mathcal{F})$ denotes the family of all Suslin- $\mathcal{F}$ sets. Also, let $\mu$ be any measure and let $\mathcal{M}$ be the class of all $\mu$-measurable sets. Then all Suslin- $\mathcal{N}$ sets are $\mu$-measurable, that is $\mathfrak{S}(\mathcal{N})=\mathcal{N}$; see $[27$, Theorem 26]. In particular, if $\mathcal{F}$ is a family of $\mu$-measurable sets then $\mathfrak{S}(\mathcal{F}) \subseteq \mathcal{M}$.

We define a metric $\sigma$ on $\mathbb{N}^{\mathbb{N}}$ by

$$
\sigma(\mathbf{i}, \mathbf{j})= \begin{cases}0 & \text { if } \mathbf{i}=\mathbf{j} \\ 2^{-\lambda} & \text { if } \mathbf{i} \neq \mathbf{j}\end{cases}
$$

where $\lambda$ is the least positive integer such that $i_{\lambda} \neq j_{\lambda}$. We note that with this metric $\mathbb{N}^{\mathbb{N}}$ is a complete separable (ultra-)metric space; the resulting topological space is often called the Baire space. 
In what follows it will be useful to denote by $\mathcal{N}$ the set of points $\mathbb{N}^{\infty} \cup \mathbb{N}^{\mathbb{N}}$ together with the partial order $\sqsubseteq$, and the resulting Scott topology, where $\lambda \sqsubseteq \kappa$ whenever either $\lambda=\kappa$ or $\lambda \in \mathbb{N}^{n}$ (for some $n \in \mathbb{N}$ ) and $\lambda=\kappa \mid n$; that is, $\kappa \in \mathbb{N}^{\mathbb{N}}$ or $\kappa \in \mathbb{N}^{m}$ with $m \geqslant n$. Notice that $\mathcal{N}$ is a domain, with $\mathbb{N}^{\infty}$ a countable basis of approximation (of algebraic elements), and with ( ) (the empty sequence) bottom. Furthermore, the inherited topology (from the Scott topology) on $\mathbb{N}^{\mathbb{N}}$ (the space of maximal points) is precisely the topology generated by the metric $\sigma$. We also note that for $\kappa \in \mathbb{N}^{\infty}$ we have

$$
(\kappa)=\kappa \uparrow \cap \mathbb{N}^{\mathbb{N}}=\kappa \uparrow \cap \mathbb{N}^{\mathbb{N}}=[\kappa] .
$$

\subsection{D-Suslin subsets}

For the remainder of this section we will be concerned with the Suslin operation using the structure of $D$ - the domain in which the space of points $X$ is embedded. We define a $D$ Suslin scheme to be a function $d: \mathbb{N}^{\infty} \rightarrow D$ such that for all $\kappa \in \mathbb{N}^{\infty}$ and all $i \in \mathbb{N}$ we have $d(\kappa) \ll d(\kappa \cdot i)$ and for all $\mathbf{i} \in \mathbb{N}^{\mathbb{N}}$ we have $\bigsqcup_{n=0}^{\infty} d(\mathbf{i} \mid n) \in e(X)$. In this way the function $d$ can be extended to a unique continuous function $\bar{d}: \mathcal{N} \rightarrow D$ such that $\bar{d}\left(\mathbb{N}^{\mathbb{N}}\right) \subseteq e(X)$. Without loss of generality we shall also impose the condition that $d(())=\perp$.

A subset $A \subseteq X$ will be said to be a $D$-Suslin set if there exists a $D$-Suslin scheme $d: \mathbb{N}^{\infty} \rightarrow D$ such that

$$
A=\bigwedge_{\kappa \in \mathbb{N}^{\infty}}[d(\kappa)]=\bigcup_{\mathbf{i} \in \mathbb{N}^{\mathbb{N}}} \bigcap_{n=0}^{\infty}[d(\mathbf{i} \mid n)]
$$

Note that for each $\mathbf{i} \in \mathbb{N}^{\mathbb{N}}$ we have

$$
\bigsqcup_{n=0}^{\infty} d(\mathbf{i} \mid n)=\bigcap_{n=0}^{\infty}[d(\mathbf{i} \mid n)]=\bigcap_{n=0}^{\infty}(d(\mathbf{i} \mid n)) .
$$

Consequently, $A=\bar{d}\left(\mathbb{N}^{\mathbb{N}}\right)$ and

$$
A=\bigwedge_{\kappa \in \mathbb{N}^{\infty}}(d(\kappa))=\bigcup_{\mathbf{i} \in \mathbb{N}^{\mathbb{N}}} \bigcap_{n=0}^{\infty}(d(\mathbf{i} \mid n)) .
$$

It follows that the set $A$ is $\mu$-measurable for any Borel measure $\mu$ on $X$, since each $(d(\mathbf{i} \mid n))$ is $\mu$-measurable.

Given a $D$-Suslin set $A$ and corresponding $D$-Suslin scheme $d$, we may decompose $A$ into a collection of smaller and smaller sets $E(\kappa)$ where $\kappa$ ranges in $\mathbb{N}^{\infty}$. We suppose that $\kappa \in \mathbb{N}^{j}$, where $j \in \mathbb{N}$. We define the $\kappa$-cylinder of $A$ to be the set of points

$$
A(\kappa)=\bigcup\left\{\bigcap_{n=j}^{\infty}[d(\mathbf{i} \mid n)] \mid \mathbf{i} \in \mathbb{N}^{\mathbb{N}} \text { and } \mathbf{i} \mid j=\kappa\right\} \text {. }
$$

Writing $\kappa \cdot \mathbf{i} \mid n$, where $\mathbf{i} \in \mathbb{N}^{\mathbb{N}}$ and $n \in \mathbb{N}$, for the concatenation of (the finite sequence) $\kappa$ followed by (the finite sequence) $\mathbf{i} \mid n$, we have

$$
A(\kappa)=\bigcup_{\mathbf{i} \in \mathbb{N}^{\mathbb{N}}} \bigcap_{n=0}^{\infty}[d(\kappa \cdot \mathbf{i} \mid n)]=\bigcup_{\mathbf{i} \in \mathbb{N}^{\mathbb{N}}} \bigcap_{n=0}^{\infty}(d(\kappa \cdot \mathbf{i} \mid n))
$$

and hence, for $\mu$ a Borel measure on $X$, the set $A(\kappa)$ is $\mu$-measurable. 
We see that

$$
A(\kappa)=\bigcup_{n=0}^{\infty} A(\kappa \cdot n) \subseteq[d(\kappa)]
$$

for all $\kappa \in \mathbb{N}^{\infty}$. However this is not a disjoint union. For $\kappa \in \mathbb{N}^{j}$, we set

$$
E(\kappa)=A(\kappa) \backslash \bigcup_{i=1}^{j} \bigcup_{n=0}^{\kappa_{i}-1} A(\kappa \mid(i-1) \cdot n),
$$

where the interpretation for $j=0$ is that $E(())=A(())=A$. It now follows that for all $\kappa \in \mathbb{N}^{\infty}$,

$$
E(\kappa)=\bigcup_{n=0}^{\infty} E(\kappa \cdot n)
$$

where the union is disjoint. Consequently, by induction, we have for $j \in \mathbb{N}$

$$
A=\bigcup_{\kappa \in \mathbb{N}^{j}} E(\kappa)
$$

again the union being disjoint.

Alternatively, it is sufficient to define $\prec$ on $\mathbb{N}^{j}$ as the lexicographical ordering, where $\lambda \prec \kappa$ whenever $\lambda \neq \kappa$ and for the least $i$ with $\lambda_{i} \neq \kappa_{i}$ we have $\lambda_{i}<\kappa_{i}$. Then for $\kappa \in \mathbb{N}^{j}$ we have

$$
E(\kappa)=A(\kappa) \backslash \bigcup_{\lambda \prec \kappa} A(\lambda)
$$

where $\lambda$ ranges in $\mathbb{N}^{j}$. The disadvantage is that the union is infinite.

\subsection{The construction of a sequence of approximating valuations}

We suppose that $A$ is a $D$-Suslin subset of $X$, with $D$-Suslin scheme $d: \mathbb{N}^{\infty} \rightarrow D$ such that $d(())=\perp$. We also suppose that $\mu$ is a $\mathcal{T}$-continuous probability Borel measure supported on $A$ (that is, $\mu(X \backslash A)=0)$ such that the quantities

$$
m(\kappa)=\mu(E(\kappa))
$$

are known for each $\kappa \in \mathbb{N}^{\infty}$. Note that $\mu(A)=1$ and hence $m(())=1$. Using the $D$-Suslin scheme $d: \mathbb{N}^{\infty} \rightarrow D$ and the function $m: \mathbb{N}^{\infty} \rightarrow \mathbb{R}$, we construct a sequence $\left(v_{n}\right)_{n=0}^{\infty}$ of simple valuations approximating $\hat{\mu}$.

We start with $\nu_{0}=\eta_{\perp}$, which in our notation may be represented as $\eta_{d(())}$. Our goal will be to choose a sequence $\left(\kappa(n) \in \mathbb{N}^{n}\right)_{n=1}^{\infty}$, with $\kappa(n)=(k(n, 1), \ldots, k(n, n))$ say, and a sequence of 'coefficient' functions $\left(r_{n}\right)_{n=0}^{\infty}$. To aid with notation we will order for $n \in \mathbb{N}$ each $\mathbb{N}^{n}$ by the pointwise order $\leqslant_{n}$, where $\lambda \leqslant_{n} \kappa$ whenever $\lambda_{i} \leqslant \kappa_{i}$ for $i=1, \ldots, n$; and of course ()$\leqslant 0()$. Then for $\kappa \in \mathbb{N}^{n}$ we will write

$$
[0, \kappa]=\left\{\lambda \in \mathbb{N}^{n} \mid \lambda \leqslant n \kappa\right\} .
$$

At the $n$th stage $r_{n}:[0, \kappa(n)] \cup\{()\} \rightarrow \mathbb{R}$ is chosen and $v_{n}$ will be defined as

$$
v_{n}=\sum_{\lambda \in[0, \kappa(n)]} r_{n}(\lambda) \eta_{d(\lambda)}+r_{n}(()) \eta_{d(())},
$$


where $r_{n}(())$ is defined by

$$
r_{n}(())=\left(1-\sum_{\lambda \in[0, \kappa(n)]} r_{n}(\lambda)\right) .
$$

Our construction will be based on the simple fact that for $n=1,2, \ldots$ we have

$$
\left(1-2^{-n}\right)^{2}=1-2^{1-n}+2^{-2 n}>1-2^{1-n} .
$$

We choose $\kappa(1)=(k(1,1))$ so that

$$
\sum_{\lambda \in[0, \kappa(1)]} m(\lambda)=\sum_{i=0}^{k(1,1)} m(() \cdot i)=\sum_{i=0}^{k(1,1)} m(i) \geqslant \frac{1}{2},
$$

(where we omit the redundant parenthesis in ' $m((i))$ '). Then set for $\lambda=(i) \in[0, \kappa(1)]$

$$
r_{1}(\lambda)=\frac{m(\lambda)}{2}=\frac{m(i)}{2} .
$$

Observe that

$$
\frac{1}{2} \geqslant \sum_{\lambda \in[0, \kappa(1)]} r_{1}(\lambda) \geqslant\left(\frac{1}{2}\right)^{2}>0 .
$$

Next, we choose $\kappa(2)=(k(2,1), k(2,2))$ with $\kappa(1) \leqslant 1 \kappa(2) \mid 1$ such that

$$
\sum_{i=0}^{k(2,1)} m(() \cdot i)=\sum_{i=0}^{k(2,1)} m(i) \geqslant \frac{7}{8},
$$

and then for each $\lambda=(i) \leqslant 1 \kappa(2) \mid 1$

$$
\sum_{j=0}^{k(2,2)} m(\lambda \cdot j)=\sum_{j=0}^{k(2,2)} m(i, j) \geqslant \frac{7 m(\lambda)}{8} .
$$

Then set for $\lambda=(i, j) \in[0, \kappa(2)]$

$$
r_{2}(\lambda)=\frac{3 m(\lambda)}{4}=\frac{3 m(i, j)}{4} .
$$

Observe that

$$
\frac{3}{4} \geqslant \sum_{\lambda \in[0, \kappa(2)]} r_{2}(\lambda) \geqslant \frac{3}{4}\left(\frac{7}{8}\right)^{2}>\frac{1}{2},
$$

and that for $\lambda \in[0, \kappa(1)]$ we have

$$
\sum_{j=0}^{k(2,2)} r_{2}(\lambda \cdot j) \geqslant \frac{3}{4}\left(\frac{7 m(\lambda)}{8}\right)>\frac{m(\lambda)}{2}=r_{1}(\lambda) .
$$

We continue in this way, so that at the $n$th stage we choose $\kappa(n)=(k(n, 1), \ldots, k(n, n))$ with $\kappa(n-1) \leqslant n-1 \kappa(n) \mid(n-1)$, such that

$$
\sum_{i=0}^{k(n, 1)} m(() \cdot i)=\sum_{i=0}^{k(n, 1)} m(i) \geqslant \frac{2^{2 n-1}-1}{2^{2 n-1}}=1-2^{1-2 n},
$$




\section{Domain-theoretic integration.}

and then for $j=1, \ldots, n-1$ (in that order) and for each $\lambda \leqslant_{j} \kappa(n) \mid j$ we have

$$
\sum_{i=0}^{k(n, j+1)} m(\lambda \cdot i) \geqslant \frac{2^{2 n-j}-1}{2^{2 n-j}} m(\lambda)=\left(1-2^{j-2 n}\right) m(\lambda) .
$$

Then set for $\lambda \in[0, \kappa(n)]$

$$
r_{n}(\lambda)=\left(1-2^{-n}\right) m(\lambda)
$$

Again, observe that

$$
1 \geqslant \sum_{\lambda \in[0, \kappa(n)]} m(\lambda) \geqslant\left(1-2^{1-2 n}\right) \prod_{j=1}^{n-1}\left(1-2^{j-2 n}\right)>\left(1-2^{-n}\right),
$$

and hence that

$$
1-2^{-n} \geqslant \sum_{\lambda \in[0, \kappa(n)]} r_{n}(\lambda)>1-2^{1-n} .
$$

Also, observe that for $\lambda \in[0, \kappa(n-1)]$ we have

$$
\sum_{i=0}^{k(n, n)} r_{n}(\lambda \cdot i) \geqslant\left(1-2^{-n}\right)\left(1-2^{-n-1}\right) m(\lambda)>\left(1-2^{1-n}\right) m(\lambda)=r_{n-1}(\lambda) .
$$

\subsection{Properties of the sequence of valuations}

Here we show that the sequence $\left(v_{n}\right)_{n=0}^{\infty}$ is such that

$$
v_{0} \ll^{1} \cdots \ll^{1} v_{n} \ll^{1} v_{n+1} \ll^{1} \cdots \ll^{1} \mu
$$

for $n=0,1, \ldots$, and that $\hat{\mu}=\bigsqcup_{n=0}^{\infty} v_{n}$. It follows that

$$
\int_{*} f \mathrm{~d} \mu=\lim _{n \rightarrow \infty} \int f_{*} \mathrm{~d} v_{n} \text { and } \int^{*} f \mathrm{~d} \mu=\lim _{n \rightarrow \infty} \int f^{*} \mathrm{~d} v_{n},
$$

and, consequently, that $f \in \mathcal{R}(\mu)$ if and only if

$$
\lim _{n \rightarrow \infty} \int f_{*} \mathrm{~d} v_{n}=\lim _{n \rightarrow \infty} \int f^{*} \mathrm{~d} v_{n}
$$

Lemma 16. Let $n \in \mathbb{N}$. Then $v_{n} \ll^{1} v_{n+1}$.

Proof. By Lemma 3 it is sufficient to define $t_{\theta, \lambda}$ for $\theta \in[0, \kappa(n)]$ and $\lambda \in[0, \kappa(n+1)]$ by

$$
t_{\theta, \lambda}= \begin{cases}0 & \text { if } \theta \neq \lambda \mid n ; \\ r_{n}(\theta) r_{n+1}(\lambda) /\left(\sum_{i=0}^{k(n+1, n+1)} r_{n+1}(\theta \cdot i)\right) & \text { if } \theta=\lambda \mid n,\end{cases}
$$

and

$$
t_{(), \lambda}= \begin{cases}r_{n+1}(\lambda) & \text { if } \lambda \mid n \notin[0, \kappa(n)] \\ r_{n+1}(\lambda)-t_{\lambda \mid n, \lambda} & \text { if } \lambda \mid n \in[0, \kappa(n)],\end{cases}
$$

and

$$
t_{\theta,()}=0 \quad \text { and } \quad t_{(),()}=r_{n+1}(()) .
$$

It is now a simple matter to show that for $\theta \in[0, \kappa(n)] \cup\{()\}$,

$$
\sum_{\lambda \in[0, \kappa(n+1)] \cup\{()\}} t_{\theta, \lambda}=r_{n}(\theta),
$$


and for $\lambda \in[0, \kappa(n+1)] \cup\{()\}$,

$$
t_{(), \lambda} \neq 0 \quad \text { and } \sum_{\theta \in[0, \kappa(n)] \cup\{()\}} t_{\theta, \lambda}=r_{n+1}(\lambda),
$$

and that $t_{\theta, \lambda} \neq 0$ implies $d(\theta) \ll d(\lambda)$.

Lemma 17. Let $n \in \mathbb{N}$. Then $v_{n} \ll \hat{\mu}$.

Proof. It is sufficient to prove that $v_{n+1} \sqsubseteq^{1} \hat{\mu}$. Let $O$ be an open subset of $D$. If $\perp \in O$ then $O=D$ and consequently

$$
v_{n+1}(O)=1=\hat{\mu}(O)=\mu\left(e^{-1}(O)\right) .
$$

Thus we may suppose that $\perp \notin O$, and define

$$
I=\{\lambda \in[0, \kappa(n+1)] \mid d(\lambda) \in O\} .
$$

We note that for each $\lambda \in I$ we have

$$
E(\lambda) \subseteq A(\lambda) \subseteq[d(\lambda)] \subseteq e^{-1}(O) .
$$

Hence

$$
v_{n+1}(O)=\sum_{\lambda \in I} r_{n+1}(\lambda)<\sum_{\lambda \in I} m(\lambda)=\mu\left(\bigcup_{\lambda \in I} E(\lambda)\right) \leqslant \mu\left(e^{-1}(O)\right)=\hat{\mu}(O),
$$

as required.

Theorem 18. We have $\hat{\mu}=\bigsqcup_{n=0}^{\infty} v_{n}$ in $\mathrm{P}^{1} D$.

Proof. Let $O$ be an open subset of $D$. It suffices to show that

$$
\lim _{n \rightarrow \infty} v_{n}(O)=\hat{\mu}(O)=\mu\left(e^{-1}(O)\right) .
$$

We consider the sets

$$
\Lambda(n)=\left\{\lambda \in \mathbb{N}^{n} \mid d(\lambda) \in O\right\} .
$$

Then for each $x \in e^{-1}(O) \cap A$ there exists $\mathbf{i} \in \mathbb{N}^{\mathbb{N}}$ such that $e(x)=\bigsqcup_{n=0}^{\infty} d(\mathbf{i} \mid n)$ and hence $d(\mathbf{i} \mid n) \in \Lambda(n)$ for all sufficiently large $n \in \mathbb{N}$. Thus

$$
e^{-1}(O) \cap A \subseteq \bigcup_{n=0}^{\infty} \bigcup_{\lambda \in \Lambda(n)} E(\lambda)
$$

Conversely, for $n \in \mathbb{N}$ and $\lambda \in \Lambda(n)$ we have $E(\lambda) \subseteq[d(\lambda)] \cap A \subseteq e^{-1}(O) \cap A$, and hence equality holds in equation (5.4). Also, for all $n \in \mathbb{N}$ we have

$$
\bigcup_{\lambda \in \Lambda(n)} E(\lambda) \subseteq \bigcup_{\lambda \in \Lambda(n+1)} E(\lambda)
$$

since if $\lambda \in \Lambda(n)$ then $\lambda \cdot i \in \Lambda(n+1)$ for all $i \in \mathbb{N}$. It follows that

$$
\hat{\mu}(O)=\mu\left(e^{-1}(O)\right)=\mu\left(e^{-1}(O) \cap A\right)=\lim _{n \rightarrow \infty} \sum\{m(\lambda) \mid \lambda \in \Lambda(n)\} .
$$


Letting $\left(\kappa(n) \in \mathbb{N}^{n}\right)_{n=1}^{\infty}$ denote the sequence used in the construction of $\left(v_{n}\right)_{n=0}^{\infty}$ to define $\left(r_{n}:[0, \kappa(n)] \rightarrow \mathbb{R}\right)_{n=1}^{\infty}$, we note that

$$
\lim _{n \rightarrow \infty} \sum\left\{m(\lambda) \mid \lambda \in \mathbb{N}^{n} \backslash[0, \kappa(n)]\right\}=0 .
$$

Thus

$$
\begin{aligned}
\lim _{n \rightarrow \infty} & \sum\left\{r_{n}(\lambda) \mid \lambda \in \Lambda(n) \cap[0, \kappa(n)]\right\} \\
& =\lim _{n \rightarrow \infty}\left(1-2^{-n}\right) \sum\{m(\lambda) \mid \lambda \in \Lambda(n) \cap[0, \kappa(n)]\} \\
& =\lim _{n \rightarrow \infty} \sum\{m(\lambda) \mid \lambda \in \Lambda(n) \cap[0, \kappa(n)]\}=\hat{\mu}(O),
\end{aligned}
$$

and the result follows.

Corollary 19. Let $f: X \rightarrow[\alpha, \beta]$ be bounded. Then

$$
\int_{*} f \mathrm{~d} \mu=\lim _{n \rightarrow \infty} \int f_{*} \mathrm{~d} v_{n} \text { and } \int^{*} f \mathrm{~d} \mu=\lim _{n \rightarrow \infty} \int f^{*} \mathrm{~d} v_{n} .
$$

Proof. For $n=0,1, \ldots$ we have $v_{n} \in S(\hat{\mu})$, and hence

$$
\int f_{*} \mathrm{~d} v_{n} \leqslant \int_{*} f \mathrm{~d} \mu \leqslant \int^{*} f \mathrm{~d} \mu \leqslant \int f^{*} \mathrm{~d} v_{n} .
$$

Conversely, for each $\lambda \in S(\hat{\mu})$, since $\hat{\mu}=\bigsqcup_{n=0}^{\infty} v_{n}$, we have $\lambda \bigsqcup^{1} v_{n}$ for all sufficiently large $n \in \mathbb{N}$. Thus, by Lemma 6, we have

$$
\int f_{*} \mathrm{~d} \lambda \leqslant \lim _{n \rightarrow \infty} \int f_{*} \mathrm{~d} v_{n} \text { and } \lim _{n \rightarrow \infty} \int f^{*} \mathrm{~d} \nu_{n} \leqslant \int f^{*} \mathrm{~d} \lambda,
$$

and the result follows.

\subsection{Quotient measures}

Here we suppose that $\mu$ is a $\mathcal{T}$-continuous Borel measure on $X$, and that $A$ is a $D$-Suslin subset of $X$ with $0<\mu(A)<\infty$. We show that our definition of $\int_{A} f \mathrm{~d} \mu$, in equation (5.2), is well defined.

Proposition 20. Let $\mu$ and $A$ be as above. Then the measure $\mu / A$, as defined in equation (5.1), is a $\mathcal{T}$-continuous probability Borel measure supported on A.

Proof. It is clear that $\mu / A$ is a probability measure supported on $A$. Thus the result will follow on showing that all Borel sets are $(\mu / A)$-measurable, $\mu / A$ is $\mathcal{B}$-regular, and $\mu / A$ is $\mathcal{T}$-continuous.

Let $M$ be a $\mu$-measurable set. Then for all $E \subseteq X$ we have $\mu(E \cap A)=\mu((E \cap A) \cap M)+\mu((E \cap A) \backslash M)=\mu((E \cap M) \cap A)+\mu((E \backslash M) \cap A)$. Thus $(\mu / A)(E)=(\mu / A)(E \cap M)+(\mu / A)(E \backslash M)$, and hence $M$ is $(\mu / A)$-measurable. Consequently, $\mathcal{B} \subseteq \mathcal{M}_{\mu} \subseteq \mathcal{M}_{\mu / A}$.

We use the $\mathcal{B}$-regularity of $\mu$ and the fact that $\mathcal{B}$ is a $\sigma$-algebra to show that $\mu / A$ is $\mathcal{B}$-regular. We recall, from Subsection 5.2, that $A$ is $\mu$-measurable since it is $D$-Suslin. Since $\mathcal{B}$ is closed under countable intersections we may choose $B_{1} \in \mathcal{B}$ such that $A \subseteq B_{1}$ and $\mu\left(B_{1}\right)=\mu(A)$. Similarly, we may choose $B_{2} \in \mathcal{B}$ such that $B_{1} \backslash A \subseteq B_{2}$ and

$$
\mu\left(B_{2}\right)=\mu\left(B_{1} \backslash A\right)=\mu\left(B_{1}\right)-\mu(A)=0 .
$$


Defining $C=B_{1} \backslash B_{2}$, we see that $C \subseteq A$ and $\mu(A \backslash C)=0$. Since $\mathcal{B}$ is closed under complements, we also have $C \in \mathcal{B}$ and $X \backslash C \in \mathcal{B}$. Thus for all $E \subseteq X$ and $B_{3} \in \mathcal{B}$ with $E \cap A \subseteq B_{3}$ we have $E \subseteq B_{3} \cup(X \backslash C)$ and

$$
(\mu / A)\left(B_{3} \cup(X \backslash C)\right)=\frac{\mu\left(\left(B_{3} \cap A\right) \cup(A \backslash C)\right)}{\mu(A)}=\frac{\mu\left(B_{3} \cap A\right)}{\mu(A)} \leqslant \frac{\mu\left(B_{3}\right)}{\mu(A)} .
$$

The $\mathcal{B}$-regularity of $\mu / A$ follows on taking the infimum over all such sets $B_{3}$.

Finally, we let $\mathcal{U}$ be a family of open subsets of $X$, which is (upwardly) directed by $\subseteq$, and let $U=\bigcup \mathcal{U}$. Letting $d: \mathbb{N}^{\infty} \rightarrow D$ be a $D$-Suslin scheme that generates $A$, we consider the set

$$
K=\left\{\kappa \in \mathbb{N}^{\infty} \mid \exists V \in \mathcal{U}: E(\kappa) \subseteq V\right\} .
$$

It is clear that $\bigcup_{\kappa \in K} E(\kappa) \subseteq U \cap A$. Conversely, for all $x \in U \cap A$ we may choose $V \in \mathcal{U}$ such that $x \in V$. Then, as in the proof of Theorem 18 , there exists $\mathbf{i} \in \mathbb{N}^{\mathbb{N}}$ such that $e(x)=\bigsqcup_{n=1}^{\infty} d(\mathbf{i} \mid n)$ and hence $E(\mathbf{i} \mid n) \subseteq[d(\mathbf{i} \mid n)] \subseteq V$ for all sufficiently large $n \in \mathbb{N}$. Thus $U \cap A=\bigcup_{\kappa \in K} E(\kappa)$. Since $K$ is countable and $\mathcal{U}$ is directed we may choose a sequence $\left(V_{n}\right)_{n=1}^{\infty}$ of sets in $\mathcal{U}$ such that $V_{n} \subseteq V_{n+1}$ for $n=1,2, \ldots$ and $U \cap A=\bigcup_{n=1}^{\infty} V_{n} \cap A$. It follows from the fact that $\mu$ is a Borel measure that $\mu(U \cap A)=\lim _{n \rightarrow \infty} \mu\left(V_{n} \cap A\right)$. Therefore,

$$
(\mu / A)(U)=\lim _{n \rightarrow \infty}(\mu / A)\left(V_{n}\right) \leqslant \sup _{V \in \mathcal{U}}(\mu / A)(V) \leqslant(\mu / A)(U),
$$

as required.

Note 3. The above proof that $\mu / A$ is $\mathcal{T}$-continuous is based on the separability (in the inherited topology) of the $D$-Suslin set $A$. An alternative, say if $A$ were an arbitrary measurable subset of $X$, would be to assume that $\mu$ is $\mathcal{T}$-regular. We may then be interested in whether $\mu / A$ is $\mathcal{T}$-regular. The proof of this can be set out in a similar fashion to the proof of $\mathcal{B}$-regularity, provided that $A$ is $\mu$-measurable and

$$
\mu(A)=\sup \{\mu(C) \mid C \subseteq A \text { and } C \text { is closed in } X\} .
$$

In the case when $\mu$ is finite, equation (5.5) follows easily from the assumptions that $\mu$ is $\mathcal{T}$-regular and $\mathcal{T} \subseteq \mathcal{M}_{\mu}$; that is, all open sets are $\mu$-measurable. If $\mu$ is not finite then this result is more problematic. However, under the assumptions that $A$ is $D$-Suslin and $\mu$ is a $\mathcal{T}$-regular Borel measure, we may by standard methods, see [16, Theorem 2.2.12], choose compact $C \subseteq A$ with $\mu(C)$ as close as we wish to $\mu(A)$, and hence equation (5.5) holds since $X$ is Hausdorff.

\section{Analytic sets}

The aim of this section is to discuss sufficient conditions under which an analytic subset $A$ of $X$ is a $D$-Suslin subset of $X$. In particular, if $X$ is a metric space and $D=\mathcal{J}(\mathbf{B} X)_{\perp}$ as described in Subsection 2.2 then every non-empty analytic subset is $D$-Suslin. We define a set to be analytic whenever it is the continuous image of a polish space; this is slightly more general than the definition given in $[\mathbf{2 0}, \mathbf{1 4}$.5]. Note that every $D$-Suslin subset $A$ of $X$ is analytic, since $A=\bar{d}\left(\mathbb{N}^{\mathbb{N}}\right)$ where $\bar{d}$ is the extension of any $D$-Suslin scheme $d: \mathbb{N}^{\infty} \rightarrow D$ that generates $A$.

We begin by recalling some of the standard results concerning analytic sets and polish spaces. We then introduce the notion of a countable graded cover of $A$ from $D$, and show 
that if $X$ is a metric space and $D=\mathcal{J}(\mathbf{B} X)_{\perp}$ then every non-empty analytic subset $A$ has a countable graded cover. The next subsection shows that every non-empty analytic subset with a countable graded covering from $D$ is $D$-Suslin. We finish this section with a discussion of the support of a $\mathcal{T}$-continuous probability Borel measure.

Note, however, that without some condition on the space $X$ we cannot expect that every analytic set is $D$-Suslin. For example, in the case when $X$ contains a point $x$ such that $A=\{x\}$ is not a countable intersection of open sets, clearly $A$ is the continuous image of the one-point space, and hence analytic. But if $d: \mathbb{N}^{\infty} \rightarrow D$ is a $D$-Suslin scheme, such that $A$ is the set resulting from the Suslin operation applied to $d$, then for each $\mathbf{i} \in \mathbb{N}^{\mathbb{N}}$ we must have $A=\{x\}=\bigcap_{n=0}^{\infty}(d(\mathbf{i} \mid n))$, contradicting our initial hypothesis.

\subsection{Preliminary results}

Throughout this section we will suppose that $A$ is a non-empty analytic subset of $X$. As is well known, every non-empty polish space is the continuous image of the Baire space $\mathbb{N}^{\mathbb{N}}$, and hence we may assume that $A$ is the image of a continuous function $g: \mathbb{N}^{\mathbb{N}} \rightarrow X$. We recall that a topological space $X$ is polish whenever it is metrisable by a complete separable metric.

In what follows we will make frequent use of the product space $\mathbb{N}^{\mathbb{N}} \times X$ endowed with the product topology. We will denote by $p_{X}$ the projection map from $\mathbb{N}^{\mathbb{N}} \times X$ onto $X$; which is continuous by definition. Note that $\mathbb{N}^{\mathbb{N}} \times X$ is Hausdorff since both $\mathbb{N}^{\mathbb{N}}$ and $X$ are. Also, in the case that $X$ has a metric $\rho$ then $\mathbb{N}^{\mathbb{N}} \times X$ may be metrised by the product metric $\sigma \times \rho$ which is defined by

$$
(\sigma \times \rho)((\mathbf{i}, x),(\mathbf{j}, y))=\max \{\sigma(\mathbf{i}, \mathbf{j}), \rho(x, y)\},
$$

where $\sigma$ is as in equation (5.3). The metric $\sigma \times \rho$ is complete whenever $\rho$ is complete (since $\sigma$ is complete) and consequently $\mathbb{N}^{\mathbb{N}} \times X$ is polish whenever $X$ is polish (since $\mathbb{N}^{\mathbb{N}}$ is also separable). We may also (topologically) embed the space $\mathbb{N}^{\mathbb{N}} \times X$ into the product domain $\mathcal{N} \times D$ where the order $\sqsubseteq$ is defined by $(\lambda, c) \sqsubseteq(\kappa, d)$ whenever $\lambda \sqsubseteq \kappa$ and $c \sqsubseteq d$. The embedding is simply given by the product map $\iota \times e$ of the inclusion map $\iota: \mathbb{N}^{\mathbb{N}} \rightarrow \mathcal{N}$ and the embedding $e: X \rightarrow D$; that is $(\mathbf{i}, x) \mapsto(\mathbf{i}, e(x))$.

In the case when $\mathcal{F}$ is the family of all closed subsets of (the Hausdorff space) $X$, we are able to characterize the Suslin- $\mathcal{F}$ sets as the projections of closed subsets of $\mathbb{N}^{\mathbb{N}} \times X$ onto $X$. To see this, we let $S$ be a Suslin-F $\mathcal{F}$ set of the form

$$
S=\bigwedge_{\kappa \in \mathbb{N}^{\infty}} F(\kappa)=\bigcup_{\mathbf{i} \in \mathbb{N}^{\mathbb{N}}} \bigcap_{n=0}^{\infty} F(\mathbf{i} \mid n) ;
$$

then $S=p_{X}(\widetilde{S})$ where

$$
\widetilde{S}=\left\{(\mathbf{i}, y) \mid \mathbf{i} \in \mathbb{N}^{\mathbb{N}} \text { and } y \in \bigcap_{n=0}^{\infty} F(\mathbf{i} \mid n)\right\},
$$

which is closed in the product topology on $\mathbb{N}^{\mathbb{N}} \times X$. Conversely, if $\widetilde{S}$ is a closed subset of $\mathbb{N}^{\mathbb{N}} \times X$ then $p_{X}(\widetilde{S})$ has the form

$$
p_{X}(\widetilde{S})=\bigwedge_{\kappa \in \mathbb{N}^{\infty}} F(\kappa)=\bigcup_{\mathbf{i} \in \mathbb{N}^{\mathbb{N}}} \bigcap_{n=0}^{\infty} F(\mathbf{i} \mid n)
$$

where for $\kappa \in \mathbb{N}^{n}$ we take $F(\kappa)$ to be the closure (in $X$ ) of the set

$$
S(\kappa)=\left\{y \in X\left|\exists \mathbf{i} \in \mathbb{N}^{\mathbb{N}}: \kappa=\mathbf{i}\right| n \text { and }(\mathbf{i}, y) \in \widetilde{S}\right\} .
$$


Observe that in the case when $X$ is polish then $\widetilde{S}$ is a closed subset of the polish space $\mathbb{N}^{\mathbb{N}} \times X$. Thus $\widetilde{S}$ is polish, and hence $S$ is analytic.

On the other hand, every analytic subset $A$ of (the Hausdorff space) $X$ is a Suslin-F set, where $\mathcal{F}$ denotes the family of all closed subsets of $X$. To see this we may suppose that $A$ is the image of the continuous function $g: \mathbb{N}^{\mathbb{N}} \rightarrow X$. Then, letting $\widetilde{A}$ be the graph of $g$, that is

$$
\widetilde{A}=\left\{(\mathbf{i}, x) \mid \mathbf{i} \in \mathbb{N}^{\mathbb{N}} \text { and } g(\mathbf{i})=x\right\},
$$

we see, since $X$ is Hausdorff, that $\widetilde{A}$ is a closed subset of $\mathbb{N}^{\mathbb{N}} \times X$. It follows that $A$ is a Suslin$\mathcal{F}$ set by the above characterization. We deduce the following (well-known) characterization of the analytic subsets of a polish space.

Theorem 21. Let $\mathcal{F}$ be the family of all closed subsets of a polish space $X$. Then the analytic subsets of $X$ are precisely the Suslin-F sets.

In particular, the family of all analytic subsets of a complete separable metric space $X$ contains all closed subsets of $X$, and hence all Borel subsets of $X$, since it is closed under all countable unions and intersections, and every open set is a countable union of closed sets. However, in general this family is not a $\sigma$-algebra of sets, and consequently is a much larger class of subsets of $X$ than the Borel family.

\subsection{Graded coverings}

We now extend the notion of a covering of $X$ from $D$, which we met in Subsection 4.2. Recall that ' $C$ is a covering of $X$ from $D$ ' means that

$$
X \subseteq \bigcup_{c \in C}(c)
$$

and for all $c \in C$ we have $(c) \neq \varnothing$. Here we wish to consider coverings of our analytic set $A \subseteq X$. We say that $C$ is a covering of $A$ from $D$ to mean that

$$
A \subseteq \bigcup_{c \in C}(c)
$$

and for all $c \in C$ we have $(c) \cap A \neq \emptyset$. By a graded covering $B$ of $A$ from $D$ we will mean that $B=\bigcup_{n=0}^{\infty} B_{n}$ is the union of a sequence of coverings $\left(B_{n}\right)_{n=0}^{\infty}$ of $A$ from $D$ with $B_{0}=\{\perp\}$, together with a function $\widehat{:} B \rightarrow D$ satisfying the following conditions:

1. $\{(\widehat{b}) \cap A \mid b \in B\}$ is a basis of the topology of $A$;

2. for all $b \in B$ we have $\widehat{b} \ll b$;

3. for $n \in \mathbb{N}$, and for $b \in B_{n}$ and $c \in B_{n+1}$, if $[b] \cap(c) \neq \emptyset$ then $\widehat{b} \ll \widehat{c}$;

4. for every $b=\bigsqcup_{n=0}^{\infty} \widehat{b}_{n}$, where $\left(b_{n}\right)_{n=0}^{\infty}$ is a sequence with $b_{n} \in B_{n}$ and $\widehat{b}_{n} \ll \widehat{b}_{n+1}$, either $[b]=\emptyset$ or $b \in e(X)$; that is, $b=e(x)$ for some $x \in X$.

Note that in the last condition we have

$$
[b]=\bigcap_{n=0}^{\infty}\left[\widehat{b}_{n}\right]=\bigcap_{n=0}^{\infty}\left(\widehat{b}_{n}\right) \text {. }
$$

The first equality follows from [1, Proposition 2.2.10], and the second from the fact that $\widehat{b}_{n} \ll \widehat{b}_{n+1}$ implies that $\left[\widehat{b}_{n}\right] \supseteq\left(\widehat{b}_{n}\right) \supseteq\left[\widehat{b}_{n+1}\right]$. The idea here is that the sequences $\left(b_{n}\right)_{n=0}^{\infty}$ with $b_{n} \in B_{n}$ and $\widehat{b}_{n} \ll \widehat{b}_{n+1}$ can be thought of as akin to Cauchy sequences in metric spaces. 
Lemma 22. Let $m, n$ be natural numbers with $m<n$, and let $b \in B_{m}$ and $c \in B_{n}$ with $[b] \cap(c) \neq \varnothing$. Then $\widehat{b} \ll \widehat{c}$.

Proof. Since $[b] \cap(c) \neq \emptyset$, we may choose $y \in[b] \cap(c)$. Then for $i=m+1, m+2, \ldots, n$, since $B_{i}$ is a covering of $A$, we may choose $b_{i} \in B_{i}$ with $y \in\left(b_{i}\right)$ so that $b_{n}=c$. It follows by property 3 above that

$$
\widehat{b} \ll \widehat{b}_{m+1} \ll \cdots \ll \widehat{b}_{n}
$$

and hence the result follows by transitivity of $\ll$.

Further to the above definition we will refer to a countable graded covering $B$ of A from $D$ whenever each of the sets $B_{n}$ is countable (infinite or finite) for all $n \in \mathbb{N}$, and hence $B=\bigcup_{n=0}^{\infty} B_{n}$ is countable. We see that $\mathbb{N}^{\infty}=\bigcup_{n=0}^{\infty} \mathbb{N}^{n}$ together with $\widehat{:} \mathbb{N}^{\infty} \rightarrow \mathcal{N}$ as the inclusion map is a countable graded covering of $\mathbb{N}^{\mathbb{N}}$ from $\mathcal{N}$. Note also that if $B$ is a countable graded covering of $A$ from $D$ then we can define the product $\mathbb{N}^{\infty} \otimes B$ by

$$
\mathbb{N}^{\infty} \otimes B=\bigcup_{n=0}^{\infty} \mathbb{N}^{n} \times B_{n},
$$

which is a countable graded covering of $\mathbb{N}^{\mathbb{N}} \times A$ from $\mathcal{N} \times D$, where

$$
(\lambda, b) \widehat{=}=(\lambda, \widehat{b}) \text {. }
$$

The next result shows the existence of countable graded coverings of an analytic subset of a general metric space. Our proof is a construction relying on Hausdorff's maximal principle.

Proposition 23. Let $X$ be a metric space with metric $\rho$, let $D=\mathcal{J}(\mathbf{B} X)_{\perp}$ and let $e=\Downarrow \circ \iota$, as described in Subsection 2.2. Suppose that $A$ is an analytic subset of $X$. Then there exists a countable graded covering $B$ of $A$ from $D$.

Proof. To aid notation we will denote by $((x, r))$ the open ball with centre $x$ and radius $r$, which we recall is the set $((x, r) \Downarrow)$. We let $B_{0}=\{\perp\}$ and $E \subseteq X$ such that $A$ is contained in the closure of $E$; for example, $E=X$ or any dense subset of $X$ or $A$. Then for $n=1,2, \ldots$ we let

$$
F_{n}=\left\{x \in E \quad\left(\left(x, 2^{-n-2}\right)\right) \cap A \neq \emptyset\right\}
$$

and apply Hausdorff's maximal principle to choose $G_{n}$, a maximal subset of $F_{n}$ satisfying the property that for all distinct points $x, y \in G_{n}$ we have $\rho(x, y) \geqslant 2^{-n-1}$. Then we set $B_{n}=\left\{\left(x, 2^{-n}\right) \downarrow \mid x \in G_{n}\right\}$ and $B=\bigcup_{n=0}^{\infty} B_{n}$, and define the function $\widehat{A}: B \rightarrow D$ by taking $\widehat{\perp}=\perp$ and for $b \in B_{n}$, with $b=\left(x, 2^{-n}\right) \downarrow$, by $\widehat{b}=\left(x, 2^{-n+2}\right) \downarrow ;$ this is well defined since $\downarrow: \mathbf{B} X \rightarrow \mathcal{J}(\mathbf{B} X)_{\perp}$ is injective. Observe that for $n=1,2, \ldots$, if $x \in A$ then there exists $y \in E$ with $x \in\left(\left(y, 2^{-n-2}\right)\right)$. Thus, by the maximality of $G_{n}$, either $y \in G_{n}$, or there exists $z \in G_{n}$ with $\rho(z, y)<2^{-n-1}$ and hence $x \in\left(\left(z, 2^{-n}\right)\right)$. It follows that $B_{n}$ is a covering of $A$ for all $n \in \mathbb{N}$. Also notice that since $G_{n}$ satisfies the above-stated property we see that

$$
\left\{\left(\left(x, 2^{-n-2}\right)\right) \mid x \in G_{n}\right\}
$$

is a disjoint family of open subsets of $X$, each with non-empty intersection with $A$, and consequently, under the inverse image of the continuous function (mapping the polish space onto $A$ ), can be at most countable. To see property 1 , we suppose that $U$ is an open subset 
of $X$ and that $x \in A \cap U$. Then we may choose $n=1,2, \ldots$ sufficiently large such that $\left(\left(x, 2^{-n+3}\right)\right) \subseteq U$, and hence, as in the above observation, we see that there exists $z \in G_{n}$ such that $x \in\left(\left(z, 2^{-n}\right)\right)$. It follows that

$$
x \in\left(\left(z, 2^{-n+2}\right)\right) \subseteq\left(\left(x, 2^{-n+3}\right)\right) \subseteq U,
$$

and property 1 follows on taking the intersection with $A$. Property 2 is an immediate consequence of the definition of $\ll$ on $\mathbf{B} X$ and the fact that approximation is preserved in the embedding of $\mathbf{B} X$ into $\mathcal{J}(\mathbf{B} X)_{\perp}$. Property 3 is immediate when $n=0$. For $n=1,2, \ldots$ and $b=\left(x, 2^{-n}\right) \Downarrow$ and $c=\left(y, 2^{-n-1}\right) \Downarrow$ we see that $[b] \cap(c) \neq \emptyset$ implies that

$$
\rho(x, y)<2^{-n}+2^{-n-1}<2^{-n+2}-2^{-n+1} .
$$

Finally, we see that property 4 follows from the fact that if $\left(b_{n}\right)_{n=0}^{\infty}$ is a sequence with $b_{n} \in B_{n}$ then writing $b_{n}=\left(x_{n}, r_{n}\right) \downarrow$ (for $n \geqslant 1$ ) we have $\lim _{n \rightarrow \infty} r_{n}=0$.

\subsection{When analytic sets are D-Suslin}

We can now give a sufficient condition for an analytic set $A$ to be a $D$-Suslin set. When this is the case, we may apply the results of Section 5 to conclude that a $\mathcal{T}$-continuous probability Borel measure supported on $A$ is approximated by a sequence of simple valuations with supremum $\hat{\mu}$.

Theorem 24. Let $A$ be a non-empty analytic subset of $X$. Suppose that $B$ is a countable graded covering of $A$ from $D$. Then $A$ is a $D$-Suslin subset of $X$.

Proof. Let $g: \mathbb{N}^{\mathbb{N}} \rightarrow X$ be a continuous function with image $A$, and let $\tilde{A}$ denote the graph of $g$, as defined in equation (6.1). Then by considering covers of $\widetilde{A} \subseteq \mathbb{N}^{\mathbb{N}} \times A$ chosen from the countable graded covering $\mathbb{N}^{\infty} \otimes B$ of $\mathbb{N}^{\mathbb{N}} \times A$ from $\mathcal{N} \times D$, we define inductively a function $(\lambda, b): \mathbb{N}^{\infty} \rightarrow \mathbb{N}^{\infty} \otimes B$ such that

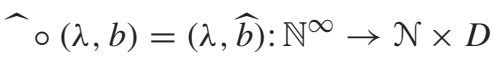

is an $(\mathcal{N} \times D)$-Suslin scheme that generates $\tilde{A}$. The result then follows by defining the $D$-Suslin scheme $d: \mathbb{N}^{\infty} \rightarrow D$ by

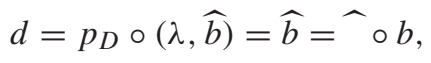

where $p_{D}$ denotes the projection map from $\mathcal{N} \times D$ onto $D$, and noting that the set resulting from the Suslin operation applied to $d$ is $A=p_{X}(\widetilde{A})$.

To define $(\lambda, b): \mathbb{N}^{\infty} \rightarrow \mathbb{N}^{\infty} \otimes B$ we start with $(\lambda(()), b(()))=((), \perp)$. Then inductively, we suppose that $(\lambda(\kappa), b(\kappa))$, where $\kappa \in \mathbb{N}^{n}$, has been chosen such that

$$
\bigcap_{m=0}^{n}([\lambda(\kappa \mid m)] \times[b(\kappa \mid m)]) \cap \widetilde{A} \neq \emptyset
$$

We let $(\lambda(\kappa \cdot i), b(\kappa \cdot i))_{i=0}^{\infty}$ be an enumeration of the set

$$
\left\{(\theta, c) \in \mathbb{N}^{n+1} \times B_{n+1} \mid((\theta) \times(c)) \cap \bigcap_{m=0}^{n}([\lambda(\kappa \mid m)] \times[b(\kappa \mid m)]) \cap \tilde{A} \neq \varnothing\right\},
$$

where we use repetition in the case when this set is finite. Note that it is non-empty since $\mathbb{N}^{n+1} \times B_{n+1}$ covers $\mathbb{N}^{\mathbb{N}} \times A$.

Observe that property 3 of a countable graded cover implies that for all $\kappa \in \mathbb{N}^{\infty}$ and all $i \in \mathbb{N}$ we have $(\lambda(\kappa), \widehat{b}(\kappa)) \ll(\lambda(\kappa \cdot i), \widehat{b}(\kappa \cdot i))$. Let $\mathbf{i} \in \mathbb{N}^{\mathbb{N}}$, and consider the corresponding 
sequence $(\lambda(\mathbf{i} \mid n), \widehat{b}(\mathbf{i} \mid n))_{n=0}^{\infty}$. By the construction, we see that $\lambda(\mathbf{i} \mid n) \ll \lambda(\mathbf{i} \mid(n+1))$ and thus $\bigsqcup_{n=0}^{\infty} \lambda(\mathbf{i} \mid n) \in \mathbb{N}^{\mathbb{N}}$. We let $x=g\left(\bigsqcup_{n=0}^{\infty} \lambda(\mathbf{i} \mid n)\right)$, and show that $x \in \bigcap_{n=0}^{\infty}[\widehat{b}(\mathbf{i} \mid n)]$. We deduce, from property 4 of a countable graded cover, that $\bigsqcup_{n=0}^{\infty} \widehat{b}(\mathbf{i} \mid n)=e(x)$. It follows that $(\lambda, \widehat{b})$ is an $(\mathcal{N} \times D)$-Suslin scheme and that

$$
\bigwedge_{\kappa \in \mathbb{N}^{\infty}}[\lambda(\kappa)] \times[\widehat{b}(\kappa)]=\bigcup_{\mathbf{i} \in \mathbb{N}^{\mathbb{N}}} \bigcap_{n=0}^{\infty}[\lambda(\mathbf{i} \mid n)] \times[\widehat{b}(\mathbf{i} \mid n)] \subseteq \tilde{A} .
$$

The converse set inclusion follows from the fact that $\widetilde{A} \subseteq(\lambda()) \times(b())$ and

$$
[\lambda(\kappa)] \times[b(\kappa)] \cap \tilde{A} \subseteq \bigcup_{i=0}^{\infty}(\lambda(\kappa \cdot i)) \times(b(\kappa \cdot i))
$$

for all $\kappa \in \mathbb{N}^{\infty}$. Thus for each $\mathbf{i} \in \mathbb{N}^{\mathbb{N}}$ there exists, by induction, a sequence $\left(\kappa_{n}\right)_{n=0}^{\infty}$, with $\kappa_{n} \in \mathbb{N}^{n}$, such that $(\mathbf{i}, g(\mathbf{i})) \in\left[\lambda\left(\kappa_{n}\right)\right] \times\left[b\left(\kappa_{n}\right)\right] \subseteq\left[\lambda\left(\kappa_{n}\right)\right] \times\left[\widehat{b}\left(\kappa_{n}\right)\right]$ for all $n \in \mathbb{N}$.

We complete the proof by showing that for $n=0,1, \ldots$ we have $x \in[\widehat{b}(\mathbf{i} \mid n)]$. Since $B_{n}$ is a cover of $A$ we may choose $c \in B_{n}$ such that $c \ll e(x)$. Then by the continuity of $g$ there exists $N \geqslant n$ such that for all $\mathbf{j} \in \mathbb{N}^{\mathbb{N}}$ with $\mathbf{j}\left|N=\lambda(\mathbf{i} \mid N)=\left(\bigsqcup_{n=0}^{\infty} \lambda(\mathbf{i} \mid n)\right)\right| N$ we have $g(\mathbf{j}) \in(c)$. But by definition we have

$$
\bigcap_{n=0}^{N}([\lambda(\mathbf{i} \mid n)] \times[b(\mathbf{i} \mid n)]) \cap \widetilde{A} \neq \emptyset,
$$

and hence $(c) \cap[b(\mathbf{i} \mid n)] \neq \emptyset$, since $N \geqslant n$. Thus, by Lemma 26 , we have

$$
\widehat{b}(\mathbf{i} \mid n) \ll \widehat{c} \ll c \ll e(x),
$$

and the result follows.

Combining this result with Proposition 23 we have the following corollary.

Corollary 25. Let $X$ be a metric space and $D=\mathfrak{J}(\mathbf{B} X)_{\perp}$ as described in Subsection 2.2. Then every analytic subset $A$ of $X$ is a D-Suslin set.

\subsection{The support of a measure in a complete metric space}

In this subsection we suppose that $X$ is a complete metric space with metric $\rho$ and that $D=\mathcal{J}(\mathbf{B} X)_{\perp}$, as discussed in Subsection 2.2. We also suppose that $\mu$ is a $\mathcal{T}$-continuous probability Borel measure on $X$. As in Subsection 6.2, we will denote by $((x, r))$ the open ball with centre $x$ and radius $r$. Recall that the support spt $\mu$ of $\mu$ may be defined by

$$
X \backslash \bigcup\{U \in \mathcal{T} \mid \mu(U)=0\},
$$

and hence $x \in \operatorname{spt} \mu$ if and only if for all open sets $U$ with $x \in U$ we have $\mu(U)>0$. We now give a construction that spt $\mu$ is $D$-Suslin; in particular, spt $\mu$ is analytic. Consequently, the results in Section 5 apply, giving a sequence of simple valuations with supremum $\hat{\mu}$. When $X$ is not complete then the result here applies in the completion $\bar{X}$ of $X$, which may be identified with the space of maximal points of $\mathcal{J}(\mathbf{B} X)_{\perp}$; in this case we may then be able to proceed further if $X$ is a $D$-Suslin subset of $\bar{X}$.

The result that $\operatorname{spt} \mu$ is analytic, for $\mu$ a finite measure on a complete metric space $X$ with all open sets $\mu$-measurable, follows easily from [16, Theorem 2.2.16], which asserts 
that spt $\mu$ is separable, and the fact that $\operatorname{spt} \mu$ is by definition closed, and hence spt $\mu$ is a complete separable metric space.

We let $E$ be any dense subset of $X$, or $E=X$, and use this to define a countable graded covering $B$ of spt $\mu$ from $D$. We proceed as in the proof of Proposition 23. For $n=1,2, \ldots$ we let

$$
F_{n}=\left\{x \in E \mid \mu\left(\left(x, 2^{-n-2}\right)\right) \neq 0\right\}
$$

and then, as before, apply Hausdorff's maximal principle to choose $G_{n}$, a maximal subset of $F_{n}$ satisfying the property that for all distinct points $x, y \in G_{n}$ we have $\rho(x, y) \geqslant$ $2^{-n-1}$. The sets $B_{n}$ and $B$, and the function $\widehat{A}: B \rightarrow D$ are also defined as in the proof of Proposition 23. Observe that by the maximality of $G_{n}$ we have

$$
\text { spt } \mu \subseteq \bigcup\left\{\left(\left(x, 2^{-n}\right)\right) \mid x \in G_{n}\right\} \text {, }
$$

and hence $B_{n}$ is a covering of spt $\mu$ for all $n \in \mathbb{N}$. Also notice that since $G_{n}$ satisfies the stated property,

$$
\left\{\left(\left(x, 2^{-n-2}\right)\right) \mid x \in G_{n}\right\}
$$

is a disjoint family of open subsets of $X$ each with positive measure, and hence can be at most countable since $\mu(X)=1$. Note that the last defining property of a graded cover follows by the completeness of $X$.


$D$-Suslin scheme that generates spt $\mu$. The definition of $b$ is by induction on $\mathbb{N}^{\infty}$ and is similar to that used in the proof of Theorem 24 . We start by letting $b(())=\perp$. Then, for each $\kappa \in \mathbb{N}^{\infty}$, we suppose that $b(\kappa)$ has been defined with $\mu((b(\kappa)))>0$. We let $(b(\kappa \cdot i))_{i=0}^{\infty}$ be an enumeration of the set

$$
\left\{c \in B_{n+1} \mid \mu((c) \cap[b(\kappa)])>0\right\},
$$

where we use repetition in the case where this set is finite. Note that it is non-empty since $B_{n+1}$ covers spt $\mu$. Since spt $\mu$ is closed and hence complete, it follows that for all $\mathbf{i} \in \mathbb{N}^{\mathbb{N}}$ we have $\bigsqcup_{n=0}^{\infty} \widehat{b}(\mathbf{i} \mid n) \in e(\operatorname{spt} \mu)$. Since $(b(\kappa \cdot i))_{i=0}^{\infty}$ covers $[b(\kappa)] \cap \operatorname{spt} \mu$, for all $\kappa \in \mathbb{N}^{\infty}$, we deduce that

$$
\operatorname{spt} \mu=\bigwedge_{\kappa \in \mathbb{N}^{\infty}}[\widehat{b}(\kappa)]=\bigcup_{\mathbf{i} \in \mathbb{N}^{\mathbb{N}}} \bigcap_{n=0}^{\infty}[\widehat{b}(\mathbf{i} \mid n)]
$$

and hence $d=\widehat{b}$ is as required. For each $\mathbf{i} \in \mathbb{N}^{\mathbb{N}}$, we may define $g(\mathbf{i})$ to be the unique $x \in X$ such that $\bigsqcup_{n=0}^{\infty} \widehat{b}(\mathbf{i} \mid n)=e(x)$. In this way we obtain a function $g: \mathbb{N}^{\mathbb{N}} \rightarrow X$ with spt $\mu=g\left(\mathbb{N}^{\mathbb{N}}\right)$, and consequently spt $\mu$ is an analytic subset of $X$.

\section{Comments}

In this section we make some comments concerning the results of this paper, and highlight certain areas for further research. Perhaps many of the statements, concerning further research, may not seem obvious and, although we give a brief sketch of a justification, we have not given complete proofs; in particular, for the justification that $f f \mathrm{~d} \mu$ recaptures the Lebesgue integral in general Hausdorff spaces. We feel that it is more appropriate that such proofs be given in any future paper that might take up these investigations. 


\subsection{Some drawbacks of the current theory}

We recall Note 2, where we remarked that the definition of the integral does not extend directly to unbounded functions. This was seen to be a direct consequence of insisting that $D$ has a least element and the relation $\ll^{1}$ in $\mathrm{P}^{1} D$. In fact, without minimal elements in $D$ then $\ll^{1}$ is easily seen to be empty, and hence $\mathrm{P}^{1} D$ is not a domain.

Perhaps the greatest drawback is that the measure $\mu$ is assumed to be a probability measure. When $\mu$ is a finite $\mathcal{T}$-continuous Borel measure, then we can renormalise and apply the construction to the measure $\mu / \mu(X)$; however, we would need to know, a priori, the precise value of $\mu(X)$. Signed measures $\mu$ can be dealt with in the case that we know the Jordan decomposition $\mu=\mu^{+}-\mu^{-}$and then apply the construction to the renormalised parts $\mu^{+}$ and $\mu^{-}$. If a theory of domain-valued valuations could be employed in the construction of the integral then it might be possible to overcome some of these disadvantages; for example, the value of $\mu(X)$ would be approximated by $v(D)$ where $v$ is a valuation taking values in say the interval domain $\mathbf{I}_{\perp} \mathbb{R}$ of $\mathbb{R}$, or even the interval domain $\mathbf{I}_{\perp} \overline{\mathbb{R}}$ of $\overline{\mathbb{R}}$.

We also remark that an important hypothesis was that $X$ is Hausdorff, and our construction is somewhat different from the construction of the integral (with respect to a valuation) on a domain as defined, for example, in [17]. When $g: D \rightarrow[\alpha, \beta]_{*}$ is Scott continuous and $\lambda \in \mathrm{P}^{1} D$ is a continuous valuation, the definition in [17] is equivalent to

$$
\int g \mathrm{~d} \lambda=\sup _{\nu \in S(\lambda)} \int g \mathrm{~d} \nu
$$

where $\int g \mathrm{~d} \nu$ is defined as in Subsection 3.1 and $S(\lambda)$ denotes the directed set

$$
\left\{v \in \mathrm{P}^{1} D \mid v \text { simple and } v \ll^{1} \lambda\right\} .
$$

Note that the simple valuations in $S(\lambda)$ allow us to approximate the value $\int g \mathrm{~d} \lambda$ from below, but not from above.

The difference between the two integrals is highlighted by the following example. Let $X$ be a Hausdorff space and $D$ a domain, as assumed throughout this paper. Let $\mu$ be the Dirac (point) mass at a point $x \in X$; that is, for $E \subseteq X$

$$
\mu(E)= \begin{cases}1 & \text { if } x \in E ; \\ 0 & \text { if } x \notin E .\end{cases}
$$

Let $d \in D$ be such that $x \in[d]$ but $x \notin(d)$. Then define $f=\mathbb{1}_{(d)}$, the characteristic function on $X$ of $(d)$, and define $g=\mathbb{1}_{d \uparrow}$, the characteristic function on $D$ of $d \uparrow$. Notice that $f=g \circ e$ and $\hat{\mu}=\eta_{e(x)}$, and that $f \notin \mathcal{R}(\mu)$, by Theorem 12, but $\int g \mathrm{~d} \hat{\mu}$ (as defined above) is well defined and takes the value that one would expect, namely 0 . However, if the Riemann-Edalat integral were to be extended to integrals on $D$, in such a way as to maintain the approximation of $\int g \mathrm{~d} \hat{\mu}$ from both above and below, then one might expect that $g$ would not be integrable.

\subsection{Variants of the functions $f_{*}$ and $f^{*}$}

Here we remark that there is some latitude in our definition of the integral in the way that we define the functions $f_{*}$ and $f^{*}$. To see this let us define for $f: X \rightarrow[\alpha, \beta]$ the following functions on $D$ with values in $[\alpha, \beta]$

$$
f_{(*)}(d)=\inf _{x \in(d)} f(x) \text { and } f^{(*)}(d)=\sup _{x \in(d)} f(x),
$$


and

$$
f_{[*]}(d)=\sup _{c \ll d} f_{(*)}(c) \quad \text { and } \quad f^{[*]}(d)=\inf _{c \ll d} f^{(*)}(c) .
$$

The next result shows that either of the two groups could replace $f_{*}$ and $f^{*}$ in the definition of the integral.

Lemma 26. Let $f: X \rightarrow[\alpha, \beta]$ and $\mu$ be a $\mathcal{T}$-continuous probability Borel measure on $X$. Then

$$
\int_{*} f \mathrm{~d} \mu=\sup _{\nu \in S(\hat{\mu})} \int f_{(*)} \mathrm{d} v=\sup _{\nu \in S(\hat{\mu})} \int f_{[*]} \mathrm{d} \nu,
$$

and similarly for the upper integral.

Proof. By the definitions of the functions $f_{(*)}, f_{*}$ and $f_{[*]}$ it is easily seen that

$$
f_{(*)} \geqslant f_{*} \geqslant f_{[*]},
$$

and hence

$$
\sup _{\nu \in S(\hat{\mu})} \int f_{(*)} \mathrm{d} \nu \geqslant \int_{*} f \mathrm{~d} \mu \geqslant \sup _{\nu \in S(\hat{\mu})} \int f_{[*]} \mathrm{d} \nu .
$$

To show equality we simply need to verify that for $v \in S(\hat{\mu})$ we have

$$
\int f_{(*)} \mathrm{d} v \leqslant \sup _{\lambda \in S(\hat{\mu})} \int f_{[*]} \mathrm{d} \lambda .
$$

By interpolation we may choose $\lambda \in S(\hat{\mu})$ such that $v \ll^{1} \lambda \ll^{1} \hat{\mu}$ and the result follows by Lemma 3 on noting that for $a, b \in D$ with $a \ll b$ we have $f_{(*)}(a) \leqslant f_{[*]}(b)$.

Finally, we remark that the functions $f_{[*]}: D \rightarrow[\alpha, \beta]_{*}$ and $f^{[*]}: D \rightarrow[\alpha, \beta]^{*}$ are continuous, and that $f=f_{[*]} \circ e$ (or $f=f^{[*]} \circ e$ ) if and only if $f$ is lower (or upper, respectively) semicontinuous; that is, the functions $f: X \rightarrow[\alpha, \beta]_{*}$ and $f: X \rightarrow[\alpha, \beta]^{*}$, respectively, are continuous.

\subsection{Questions of measure theory}

We begin by defining a measure $\mu$ on a topological space $X$ to be locally finite if for all points $x \in X$ there exists an open set $T$ such that $x \in T$ and $\mu(T)<\infty$. We may then ask whether Lemma 1 holds with finite $\mathcal{T}$-continuous Borel measure replaced by locally finite $\mathcal{T}$-continuous Borel measure.

The next question that arises comes from Subsection 5.5. We say that a measure $\mu$ is hereditary $\mathcal{T}$-continuous if for all $E \subseteq X$ and for all $\mathcal{U} \subseteq \mathcal{T}$ which are (upwardly) directed by $\subseteq$, we have

$$
\mu(E \cap \bigcup \mathcal{U})=\sup \{\mu(E \cap U) \mid U \in \mathcal{U}\} .
$$

We are interested in what natural conditions on a $\mathcal{T}$-continuous Borel measure $\mu$ imply that $\mu$ is hereditary $\mathcal{T}$-continuous. We recall that Note 3 remarked that equation (7.1) holds if $\mu$ is $\mathcal{T}$-regular and $E$ is a measurable set with $\mu(E)<\infty$. In fact, the condition that $E$ be $\mu$-measurable is irrelevant to equation (7.1); we required $E$ to be $\mu$-measurable in Subsection 5.5 to ensure that $\mu / E$ is a Borel measure. Consequently, we define a measure $\mu$ to be full whenever for all subsets $E$ of $X$ we have

$$
\mu(E)=\sup \{\mu(E \cap M) \mid M \text { is } \mu \text {-measurable and } \mu(M)<\infty\} .
$$


One may then readily verify that a $\mathcal{T}$-regular, $\mathcal{T}$-continuous full Borel measure is hereditary $\mathcal{T}$-continuous. This then raises the question of whether a $\mathcal{T}$-regular, $\mathcal{T}$-continuous locally finite Borel measure is full.

We now introduce two more concepts that will be useful in the next subsection. We will say that $\mu$ is a regular Borel measure on $X$ whenever $\mu$ is a Borel measure on $X$ which is $\mathcal{T}$-regular and satisfies the following inner regularity condition: for all $\mu$-measurable subsets $M$ of $X$ we have

$$
\mu(M)=\sup \{\mu(C) \mid C \subseteq M \text { and } C \text { is closed in } X\} .
$$

Similarly, we say that $\mu$ is a Radon-Borel measure whenever $\mu$ is a $\mathcal{T}$-regular Borel measure that satisfies the Radon condition: for all $\mu$-measurable subsets $M$ we have

$$
\mu(M)=\sup \{\mu(K) \mid K \subseteq M, K \text { is compact and } \mu(K)<\infty\} .
$$

If $\mu$ is a Radon-Borel measure and $\mathcal{U}$ is a directed (by $\subseteq$ ) family of open sets then for all compact $K \subseteq \bigcup \mathcal{U}$ there exists $U \in \mathcal{U}$ with $K \subseteq U$, since $\mathcal{U}$ was assumed to be directed. Consequently, every Radon-Borel measure is $\mathcal{T}$-continuous.

We remark that for a 'Radon measure' most authors also require that $\mu(K)<\infty$ for all compact $K$; in our terminology, this would be a locally finite Radon-Borel measure. Also, our definition of a regular Borel measure is non-standard; this term is usually used in the literature as an equivalent term for 'Radon measure'. Perhaps the most interesting question that arises here is the identification of the topological spaces for which it is true that every $\mathcal{T}$-regular, $\mathcal{T}$-continuous locally finite Borel measure (or even, every locally finite full regular Borel measure) is Radon-Borel.

Finally, we observe that a reason why complete separable metric spaces may be viewed as a natural setting for topological measure theory is that every (locally) finite Borel measure is Radon-Borel. We note that $\mathcal{T}$-continuity follows from the fact that the topology is second countable. The fact that every closed set is a countable intersection of open sets gives us $\mathcal{T}$-regularity. Lastly the Radon condition follows from the theory of analytic sets and [16, Theorem 2.2.12]; see also [23, Theorem 9.9].

\subsection{Another integral}

In this subsection we define a new integral. Our definition is akin to the Bourbaki extension that was sketched at the end of Subsection 4.4. However we work with two families of functions which, in general, will not be Stonian lattices as defined in Subsection 4.4. One family will be used to define an upper integral, and the other to define a lower integral. In fact, the functions in these families will be defined on the domain $D$ into which our Hausdorff space $X$ is embedded. The integral defined on these families that we extend will be that of integration with respect to a continuous valuation as defined, for example, in [17].

In order to relate this integral to the Lebesgue integral, we shall assume throughout this subsection that $\mu$ is a $\mathcal{T}$-continuous locally finite regular Borel measure. This gives rise to the continuous valuation $\hat{\mu} \in \mathbf{V} D$; the family of all continuous valuations which take values in $\overline{\mathbb{R}}_{0}^{+}$. The order $\sqsubseteq$ on $\mathbf{V} D$ is defined, as in Subsection 2.4 , by $v \sqsubseteq \lambda$ whenever $v(O) \leqslant \lambda(O)$ for all Scott open sets $O$. Notice, however, that this partial order induces a way below relation $\ll$ which is quite distinct from the way below relation $\ll^{1}$ on $\mathrm{P}^{1} D$. Simple valuations are defined, as before, to be finite linear combinations of point valuations; for example

$$
\sum_{a \in A} r_{a} \eta_{a} \quad \text { where } A \subseteq D \text { is finite and } \sum_{a \in A} r_{a}<\infty \text {. }
$$




\section{Domain-theoretic integration.}

We shall denote by $\bar{S}(\hat{\mu})$ the directed set

$$
\{v \in \mathbf{V} D \mid v \text { is simple and } v \ll \hat{\mu}\} .
$$

A detailed account of this theory, given in a more general setting, may be found in [17]. We remark that in the case that $\mu$ is a probability measure then we may work in $\mathrm{P}^{1} D$, replacing $\bar{S}(\hat{\mu})$ with $S(\hat{\mu})$ in what follows.

We denote by $\overline{\mathbb{R}}_{*}$ the domain of extended real numbers ordered by the usual ordering $\leqslant$, and dually $\overline{\mathbb{R}}^{*}$ ordered by $\leqslant{ }^{\mathrm{OP}}=\geqslant$. A function $g$ is said to be bounded lower simple on $D$ whenever $g: D \rightarrow \overline{\mathbb{R}}_{*}$ is continuous (with respect to the Scott topology),

$$
\text { Image }(g)=\{g(d) \mid d \in D\} \subseteq \mathbb{R}
$$

is a finite set, and

$$
\int g \mathrm{~d} \hat{\mu}=\sup _{\nu \in \bar{S}(\hat{\mu})} \int g \mathrm{~d} \nu<\infty
$$

where $\int g \mathrm{~d} v$ is defined for $v=\sum_{a \in A} r_{a} \eta_{a}$, as in Subsection 4.2, by

$$
\int g \mathrm{~d} v=\sum_{a \in A} r_{a} g(a)
$$

We denote the family of all bounded lower simple functions on $D$ by $\mathcal{F}_{*}$. Similarly, we define bounded upper simple functions on $D$ and the family $\mathcal{F}^{*}$ with $\overline{\mathbb{R}}_{*}$ replaced by $\overline{\mathbb{R}}^{*}$ and the condition (7.2) replaced by its dual; that is,

$$
\int g \mathrm{~d} \hat{\mu}=\inf _{v \in \bar{S}(\hat{\mu})} \int g \mathrm{~d} \nu>-\infty,
$$

where the infimum may be regarded as a supremum with respect to $\leqslant$ OP .

We let $\mathcal{F}^{\#}$ denote the closure of $\mathcal{F}_{*}$ under directed suprema; that is, $h \in \mathcal{F}^{\#}$ whenever there exists an upwardly directed subfamily $\mathcal{G} \subseteq \mathcal{F}_{*}$ such that $\mathcal{G} \nearrow h$. Notice that

$$
\mathcal{F}^{\#} \subseteq\left[D \rightarrow \overline{\mathbb{R}}_{*} \backslash\{-\infty\}\right],
$$

the space of all Scott continuous functions mapping $D$ into $\overline{\mathbb{R}}_{*} \backslash\{-\infty\}$, and that $\int \cdot \mathrm{d} \hat{\mu}$ may be defined on $\mathcal{F}^{\#}$ by

$$
\int h \mathrm{~d} \hat{\mu}=\sup _{g \in \mathcal{G}} \int g \mathrm{~d} \hat{\mu}=\sup _{g \in \mathcal{G}} \sup _{\nu \in \bar{S}(\hat{\mu})} \int g \mathrm{~d} \nu,
$$

for all $h \in \mathcal{F}^{\#}$. Dually, we define $\mathcal{F}_{\#}$ as the closure of $\mathcal{F}^{*}$ under directed infima; that is, directed suprema with respect to $\leqslant{ }^{\mathrm{OP}}$. We also extend $\int \cdot \mathrm{d} \hat{\mu}$ to $\mathcal{F}_{\#}$ by

$$
\int h \mathrm{~d} \hat{\mu}=\inf _{g \in \mathcal{G}} \int g \mathrm{~d} \hat{\mu}=\inf _{g \in \mathcal{G}} \inf _{\nu \in \bar{S}(\hat{\mu})} \int g \mathrm{~d} \nu,
$$

whenever $\mathcal{G} \searrow h$.

Then for $f: X \rightarrow \overline{\mathbb{R}}$ we say that $h: D \rightarrow \overline{\mathbb{R}}_{*}$ is a majorant of $f$ if $h \in \mathcal{F}^{\#}$ and $f \leqslant h \circ e$, and we denote the family of all majorants of $f$ by $\mathcal{F}^{\#}(f)$. Similarly, $h: D \rightarrow \overline{\mathbb{R}}^{*}$ is a minorant of $f$ if $h \in \mathcal{F}_{\#}$ and $f \geqslant h \circ e$, and we denote the family of all minorants of $f$ by $\mathcal{F}_{\#}(f)$. We define

$$
\int^{\#} f \mathrm{~d} \mu=\inf _{h \in \mathcal{F}^{\#}(f)} \int h \mathrm{~d} \hat{\mu} \quad \text { and } \quad \int_{\#} f \mathrm{~d} \mu=\sup _{h \in \mathcal{F}_{\#}(f)} \int h \mathrm{~d} \hat{\mu} .
$$


Whenever

$$
\int^{\#} f \mathrm{~d} \mu=\int_{\#} f \mathrm{~d} \mu \neq \pm \infty
$$

we define

$$
f f \mathrm{~d} \mu
$$

to be the common value, and say that $f$ is $\mu$-summable. We denote by $\AA(\mu)$ the family of all $\mu$-summable functions on $X$. Observe that the assumption that $\mu$ is a regular Borel measure implies that $\mathcal{F}_{*}$ and $\mathcal{F}^{*}$ are contained in $\mathcal{E}(\mu)$.

For the final extension, we let $\AA_{+}(\mu)$ denote the family of all functions $f: X \rightarrow \overline{\mathbb{R}}$ such that $f \wedge(g \circ e) \in \mathcal{E}(\mu)$ for all $g \in \mathcal{F}_{*}$, and dually $\mathcal{E}_{-}(\mu)$ contains all functions such that $f \vee(g \circ e) \in \mathcal{E}(\mu)$ for all $g \in \mathcal{F}^{*}$. We can then extend $f \cdot \mathrm{d} \mu$ to $\bar{\AA}(\mu)=\mathcal{E}_{+}(\mu) \cup \mathcal{E}_{-}(\mu)$ as in Subsection 4.4 upon checking that $\mathcal{L}(\mu)=\mathcal{E}_{+}(\mu) \cap \mathcal{L}_{-}(\mu)$.

Notice that when $X$ is completely regular, $\mu$ is a finite $\mathcal{T}$-continuous measure and $\mathcal{S}$ is the family of all bounded continuous functions $g: X \rightarrow \mathbb{R}$, as in Subsection 4.4, we have

$$
\mathcal{F}^{\#}(f)=\left\{g_{[*]} \mid f \leqslant g \in \mathcal{S}^{*}\right\} \quad \text { and } \quad \mathcal{F}_{\#}(f)=\left\{g^{[*]} \mid f \geqslant g \in \mathcal{S}_{*}\right\} .
$$

Thus, on performing the Bourbaki extension of $I: S \rightarrow \mathbb{R}$, defined by $I(f)=\int f \mathrm{~d} \mu$, we have

$$
I^{\#}(f)=\int^{\#} f \mathrm{~d} \mu \quad \text { and } \quad I_{\#}(f)=\int_{\#} f \mathrm{~d} \mu
$$

for all $f: X \rightarrow \overline{\mathbb{R}}$. Hence $\mathcal{E}(\mu)=\mathcal{L}(I)$ and $f f \mathrm{~d} \mu=\mathbf{I}(f)$ for all $f \in \mathcal{E}(\mu)$. It remains to be verified that $\overline{\mathcal{L}}(\mu)=\overline{\mathcal{L}}(I)$, and that the extension of $f \cdot \mathrm{d} \mu$ to $\overline{\mathcal{L}}(\mu)$ is given by $\overline{\mathbf{I}}$.

To see that the integral $f \cdot \mathrm{d} \mu$ recaptures the Lebesgue integral in general Hausdorff spaces, we make the following definitions. We let $\mathcal{M}$ denote the Stonian lattice of all measurable functions $f: X \rightarrow \mathbb{R}$ such that Image $(f)$ is a finite set and $\mu\left(f^{-1}(\mathbb{R} \backslash\{0\})\right)$ is finite. We define the positive Daniell integral $J: \mathcal{M} \rightarrow \mathbb{R}$ by

$$
J(f)=\sum\left\{t \mu\left(f^{-1}(t)\right) \mid t \in \operatorname{Image}(f) \text { and } t \neq 0\right\} .
$$

Then, as in [26, Chapter 12], we let $\overline{\mathbf{J}}: \overline{\mathcal{L}}(J) \rightarrow \overline{\mathbb{R}}$ be the Daniell extension of $J$. By [26, Exercise 12-7, Theorems 12.17 and 12.19 , and Corollary 12.21], we find that $\overline{\mathbf{J}}$ is precisely the Lebesgue integral with respect to $\mu$. We denote by $\mathcal{M}^{*}$ the family of all functions $g$ on $X$ which are the supremum of an increasing sequence $\left(f_{i}\right)_{i=1}^{\infty}$ in $\mathcal{M}$, and by $J^{*}$ the Daniell extension of $J$ to $\mathcal{M}^{*}$. We note for $f: X \rightarrow \overline{\mathbb{R}}$ that for all $h \in \mathcal{F}^{\#}(f)$ with $\int h \mathrm{~d} \hat{\mu}<\infty$ we have $h \in \mathcal{M}^{*}$ and $\int h \mathrm{~d} \hat{\mu}=\mu(h)=J^{*}(h)$, and hence

$$
\int^{\#} f \mathrm{~d} \mu \geqslant \inf _{g \in \mathcal{M}^{*}} J^{*}(g) .
$$

Conversely, one readily verifies, using the assumption that $\mu$ is a regular Borel measure, that for all $g \in \mathcal{M}^{*}$ with $J^{*}(g)<\infty$ and $\varepsilon>0$ there exists $h \in \mathcal{F}^{\#}(g)$ such that

$$
\mu(g)=J^{*}(g) \leqslant \int h \mathrm{~d} \hat{\mu}<\mu(g)+\varepsilon,
$$

and hence for $f: X \rightarrow \overline{\mathbb{R}}$ we have

$$
\int^{\#} f \mathrm{~d} \mu \leqslant \inf _{g \in \mathcal{M}^{*}} J^{*}(g) .
$$


Thus, as in the preceding paragraph, $£(\mu)=\mathcal{L}(J)$ and $f f \mathrm{~d} \mu=\mathbf{J}(f)$ for all $f \in \mathcal{E}(\mu)$.

In comparison to the inner extension theory of an elementary integral developed in [23, Chapter V], we denote by $E$ the family of all bounded upper semicontinuous functions $g: X \rightarrow[0, \infty)^{*}$ satisfying the condition that $\int g^{[*]} \mathrm{d} \hat{\mu}<\infty$. We define $I: E \rightarrow \mathbb{R}$ by $I(g)=\int g^{[*]} \mathrm{d} \hat{\mu}$. Then, using the notation and methods of [23], we note that $E=E_{\tau}$ and $I=I_{\tau} \mid E$, and that $\mathfrak{T}(E)$ is the family of all closed sets $C \subseteq X$ with $\mu(C)<\infty$. Hence, upon checking the condition that for all $u \leqslant v$ in $E$, we have

$$
I(v)-I(u) \leqslant \sup \{I(g) \mid g \in E \text { and } g \leqslant v-u\},
$$

the main theorem of [23, Theorem 15.9] is applicable, and thus $I$ has a unique maximal $\tau$ representation $\Delta_{\tau} \mid \mathfrak{C}\left(\Delta_{\tau}\right)$. Furthermore we have

$$
I_{\tau}(f)=\int_{*} f \mathrm{~d} \Delta_{\tau} \mid \mathfrak{C}\left(\Delta_{\tau}\right) \quad \text { for all } f \in[0, \infty]^{X} .
$$

To see that inequality (7.3) holds, we note that

$$
\sup \{I(g) \mid g \in E \text { and } g \leqslant v-u\}=I_{*}(v-u)=\int_{\#}(v-u) \mathrm{d} \mu
$$

since $v-u \geqslant 0$. Also $v, u \in \mathcal{L}(\mu)=\mathcal{L}(J)$, since $I(v)<\infty$ and $\mu$ is a regular Borel measure, and hence

$$
I(v)-I(u)=I_{*}(v)-I_{*}(u)=\mathbf{J}(v)-\mathbf{J}(u)=\mathbf{J}(v-u)=\int_{\#}(v-u) \mathrm{d} \mu .
$$

Let $\mathfrak{M}$ denote the family of all $\mu$-measurable sets; then, upon checking that $\mu$ is inner regular $\mathfrak{T}(E)$ at $\mathfrak{M}$, it follows that $\mu\left|\mathfrak{M}=\Delta_{\tau}\right| \mathfrak{M}$.

We note that in the case where $\mu$ is a locally finite Radon-Borel measure, it would be more natural to work with $E=\mathrm{USCK}^{+}(X)$, the space of all upper semicontinuous functions from $X$ to $\mathbb{R}_{0}^{+}$with compact support; see [23, Theorem 16.7] for an extended Riesz representation theorem in this setting. Also, in the case where $\mu$ is a $\mathcal{T}$-regular, $\mathcal{T}$-continuous locally finite Borel measure, it may be profitable to develop an outer extension theory of an elementary integral which should be dual to the inner theory of [23, Chapter V].

Finally, we comment that one way to give this process a computable framework, is to determine for which functions $f: X \rightarrow \mathbb{R}$ there is an effective construction of upwardly directed families $\mathcal{G}$ of bounded upper simple functions that produce majorants $g$ of $f$ and downwardly directed families $\mathcal{H}$ of bounded lower simple functions that produce minorants $h$ of $f$, such that the integrals $\int g \mathrm{~d} \hat{\mu}$ and $\int h \mathrm{~d} \hat{\mu}$ can be determined to within any desired accuracy.

\subsection{Computability}

Our first notion of computability is that of an effective representation of the Hausdorff space $X$. Given the framework of this paper, it is perhaps most natural to take $X$ embedded in an effectively given domain $D$ in the sense given in [14, Section 3]; that is, $D$ is an $\omega$-continuous pointed domain with a countable basis $C=\left\{c_{n} \mid n \in \mathbf{N}\right\}$ of approximation, such that the relation $c_{n} \ll c_{m}$ is recursively enumerable in $n, m$. Then we will say that $X$ has an effective structure whenever there is a graded covering $B=\bigcup_{n=0}^{\infty} B_{n} \subseteq C$, where each $B_{n}$ is recursively enumerable (as a subset of $C$ ), together with a computable function $\widehat{:} B \rightarrow C$, and a computable function $b: \mathbb{N}^{\infty} \rightarrow B$ such that $b\left(\mathbb{N}^{n}\right) \subseteq B_{n}$ for all $n \in \mathbb{N}$ 
and $\widehat{b}: \mathbb{N}^{\infty} \rightarrow C$ is a $D$-Suslin scheme that generates $X$; that is,

$$
X=\bigwedge_{\kappa \in \mathbb{N}^{\infty}}[\widehat{b}(\kappa)]=\bigcup_{\mathbf{i} \in \mathbb{N}^{\mathbb{N}}} \bigcap_{n=0}^{\infty}[\widehat{b}(\mathbf{i} \mid n)]
$$

Note that this implies that $X$ is separable and analytic; that is, the continuous image of a polish space.

An alternative to assuming the existence of the computable function $b: \mathbb{N}^{\infty} \rightarrow B$ is to assume that the relation $(b) \cap[c] \neq \varnothing$ is recursively enumerable. However, in this case we cannot construct the entire function $b$ (as this would require determining whether certain sets are finite or infinite), but we can construct a sequence of functions $\left(b_{n}: N_{n} \rightarrow\right.$ $B)_{n=0}^{\infty}$, where $N_{n} \subseteq N_{n+1} \subseteq \mathbb{N}^{\infty}$ and $b_{n+1} \mid N_{n}=b_{n}$ for $n \in \mathbb{N}$, which are sufficient to construct the sequence $\left(v_{n}\right)_{n=0}^{\infty}$ of simple valuations approximating $\mu$ of Subsection 5.3. We omit the details as we feel that this slight increase in generality does not merit the further complications to the construction of the sequence $\left(v_{n}\right)_{n=0}^{\infty}$. This alternative should allow us, in the case when $X$ is complete, to relate our notion of an effective representation of $X$ with the notion of an effectively given (complete) metric space, given in [15, Subsection 3.2].

The above then naturally leads to the following definition. A measure $\mu$ is effective whenever there exists a computable sequence of partial functions $\left(r_{n}: \mathbb{N}^{\infty} \rightarrow[0,1]\right)_{n=0}^{\infty}$ such that for each $n \in \mathbb{N}$ the set $\left\{\kappa \in \mathbb{N}^{\infty} \mid r_{n}(\kappa)\right.$ is defined $\}$ is finite (and computable) and the sequence of valuations $v_{n}=\sum_{\kappa \in \mathbb{N} \infty} r_{n}(\kappa) \eta_{\widehat{b}(\kappa)}$ forms an approximating sequence in $\mathrm{P}^{1} D$ with supremum $\hat{\mu}$. Our viewpoint is that we start with the sequences $\left(r_{n}\right)_{n=0}^{\infty}$ and consider the resultant measures; alternatively, in considering a computational problem involving a particular measure $\mu$, the problem itself gives a method for computing the sequence $\left(r_{n}\right)_{n=0}^{\infty}$.

For an effective $\mathcal{T}$-continuous probability Borel measure $\mu$ and bounded function $f \in$ $\mathcal{R}(\mu)$ we need to be able to approximate the values of $f_{*}(\widehat{b}(\kappa))$ and $f^{*}(\widehat{b}(\kappa))$, for all $\kappa \in \mathbb{N}^{\infty}$ such that $r_{n}(\kappa)$ is defined for some $n \in \mathbb{N}$, so that the value of $\int f \mathrm{~d} \mu$ can be evaluated to any given accuracy. Thus we may consider all bounded functions $f: X \rightarrow \mathbb{R}$ such that there exists a computable increasing sequence of computable increasing functions $\left(g_{n}: \widehat{B} \rightarrow\right.$ $\left.\overline{\mathbb{R}}_{*}\right)_{n=0}^{\infty}$ and a computable decreasing sequence of computable decreasing functions (that is, increasing with respect to $\left.\leqslant^{\mathrm{OP}}\right),\left(h_{n}: \widehat{B} \rightarrow \overline{\mathbb{R}}^{*}\right)_{n=0}^{\infty}$, where $\widehat{B}=\{\widehat{b} \mid b \in B\}$, such that for all $b \in B$

$$
g_{n}(\widehat{b}) \leqslant f_{*}(\widehat{b}) \quad \text { and } \quad f^{*}(\widehat{b}) \leqslant h_{n}(\widehat{b})
$$

and

$$
\int\left(h_{n}-g_{n}\right) \mathrm{d} v_{n}=\sum_{\kappa \in \mathbb{N} \infty} r_{n}(\kappa)\left(h_{n}(\widehat{b}(\kappa))-g_{n}(\widehat{b}(\kappa))\right) \rightarrow 0 .
$$

as $n \rightarrow \infty$. Such functions $f$ may be termed $\mu$-almost effective functions. The results of this paper imply that the value of $\int f \mathrm{~d} \mu$ exists and can be computed to any accuracy, given that $\mu$ is an effective $\mathcal{T}$-continuous probability Borel measure, and that $f$ is a $\mu$-almost effective function. An interesting question that arises is whether all bounded functions $f: X \rightarrow \mathbb{R}$, which are continuous $\mu$-almost everywhere, are $\mu$-almost effective, where $X$ has an effective representation and $\mu$ is an effective $\mathcal{T}$-continuous probability Borel measure.

That an analytic set $A \subseteq X$ is effective means there is a computable function $b: \mathbb{N}^{\infty} \rightarrow B$ such that $b\left(\mathbb{N}^{n}\right) \subseteq B_{n}$ for all $n \in \mathbb{N}$ and $\widehat{b}: \mathbb{N}^{\infty} \rightarrow C$ is a $D$-Suslin scheme that generates $A$. Then for a $\mathcal{T}$-continuous Borel measure $\mu$ on $X$ such that $0<\mu(A)<\infty$ and the function $m: \mathbb{N}^{\infty} \rightarrow[0,1]$ is computable, where $m(\kappa)=\mu(E(\kappa)) / \mu(A)$ and $E(\kappa)$ is as defined in Subsection 5.2, we can define the sequence of valuations $\left(v_{n}\right)_{n=0}^{\infty}$ as in Subsection 5.3. 
In this way we see that $\mu / A$ is an effective $\mathcal{T}$-continuous probability Borel measure. We also note that a slight increase in generality is afforded by considering sequences of partial functions $\left(b_{n}: N_{n} \rightarrow B\right)$ where $N_{n} \subseteq N_{n+1} \subseteq \mathbb{N}^{\infty}$ and $b_{n+1} \mid N_{n}=b_{n}$ for $n \in \mathbb{N}$.

Again, one may ask questions about how the definition of an effective analytic set can be weakened, say by using sequences of computable functions $\left(b_{n}\right)_{n=0}^{\infty}$, which in some sense approximate the continuous function $b: \mathbb{N}^{\infty} \rightarrow B$ satisfying the conditions stated above. We presume that such questions may be highly related to the mathematical subject of effective descriptive set theory.

\section{References}

1. S. Abramsky and A. Jung, 'Domain theory', Handbook of logic in computer science, Vol. III (ed. S. Abramsky, D. M. Gabbay and T. S. E Maibaum, Oxford University Press, 1994). 233, 235, 235, 260

2. A. Edalat, 'Domain of computation of a random field in statistical physics', Theory and Formal Methods 1994: Proceedings of the Second Imperial College Workshop (Imperial College Press, 1995). 230

3. A. Edalat, 'Domain theory and integration', Theoret. Comput. Sci. 151 (1995) 163 193. 229, 229, 229, 230, 230, 230, 232, 233, 234, 234, 238, 238, 238, 238, 239, 239, 239, 239, 239, 239, 239, 240, 240, 240, 241, 244, 244

4. A. Edalat, 'Domain theory in stochastic processes', Proc. Tenth Annual IEEE Symposium on Logic in Computer Science (LICS) (IEEE, 1995). 230

5. A. Edalat, 'Dynamical systems, measures and fractals via domain theory', Inform. and Comput. 120 (1995) 32-48. 230, 230, 234, 251

6. A. Edalat, 'Power domains and iterated function systems', Inform. and Comput. 124 (1996) 182-197. 230, 250

7. A. Edalat, 'Domains for computation in mathematics, physics and exact real arithmetic', Bull. Symbolic Logic 3 (1997) 401-452. 230

8. A. Edalat, 'When Scott is weak on the top', Math. Structures Comput. Sci. 7 (1997) 401-417. 230, 235, 235, 235

9. A. Edalat and M. H. Escardó, 'Integration in real PCF', Proc. Eleventh Annual IEEE Symposium on Logic in Computer Science (LICS) (IEEE, 1996). 230, 235

10. A. Edalat and R. Heckmann, 'A computational model for metric spaces', Theoret. Comput. Sci. 193 (1998) 53-73. 230, 230, 230, 230, 232, 235, 235, 251

11. A. EdAlat and M. KRzNARIć, 'Numerical integration with exact real arithmetic', $A u$ tomata, languages and programming, Lecture Notes in Comput. Sci. 1644 (Springer, 1999) 90-104. 230

12. A. Edalat and S. NegRi, 'The generalised Riemann integral on locally compact spaces', Topology Appl. 89 (1998) 121-150. 229, 229, 230, 230, 230, 233, 234, 234, 244, 244, 244

13. A. Edalat and J. Parry, 'An algorithm to estimate the Hausdorff dimension of selfaffine sets', Electronic Notes in Theoret. Comput. Sci. 13 (1998). 230

14. A. Edalat and P. Sünderhauf, 'A domain-theoretic approach to computability on the real line', Theoret. Comput. Sci. 210 (1997) 73-98. 230, 234, 270 
15. A. Edalat and P. Sünderhauf. 'Computable Banach spaces via domain theory', Theoret. Comput. Sci. 219 (1999) 169-184. 230, 271

16. H. Federer, Geometric measure theory, Grundlehren Math. Wiss. 153 (SpringerVerlag, 1969). 244, 246, 248, 248, 249, 249, 249, 251, 258, 263, 267

17. R. Heckmann, 'Spaces of valuations', Proc. Summer Conference on General Topology and Applications 1995, Ann. New York Acad. Sci. Vol. 806 (1996) 174-200. 230, 238, 265, 265, 267, 268

18. R. Heckmann, 'Approximation of metric spaces by partial metric spaces', Applied Categ. Structures 7 (1999) 71-83. 230, 235

19. C. Jones, 'Probabilistic non-determinism', PhD thesis, University of Edinburgh, 1989. 230,238

20. A. S. Kechris, Classical descriptive set theory, Grad. Texts in Math. 156 (SpringerVerlag, 1995). 258

21. J. L. Kelley, General topology (Van Nostrand, 1955). 233

22. O. KIRCH, 'Bereiche und Bewertungen', Master's thesis, Technische Hochschule Darmstadt, 1993. 230, 238

23. H. KöNIG, Measure and integration (Springer-Verlag, 1997). 267, 270, 270, 270, 270,270

24. J. D. Lawson, 'Spaces of maximal points', Math. Structures Comput. Sci. 7 (1997) 543-555. 230, 235, 235

25. J. D. Lawson, 'Computation on metric spaces via domain theory', Topology Appl. 85 (1998) 247-263. 230, 235, 235

26. W. F. Pfeffer, Integrals and measures, Pure Appl. Math. 42 (Dekker, 1977). 236, 246, 246, 246, 246, 250, 250, 250, 250, 269, 269

27. C. A. Rogers, Hausdorff measures (Cambridge University Press, 1970). 236, 236, 251

J. D. Howroyd j.howroydegold.ac.uk http://homepages.gold.ac.uk/jhowroyd/

Department of Mathematics

Goldsmiths College

University of London

New Cross

London SE14 6NW 